\title{
Factors associated with regular consumption of obesogenic foods: National School-Based Student Health Hurvey, 2012
}

\section{Fatores associados ao consumo regular de alimentos}

obesogênicos: Pesquisa Nacional

de Saúde do Escolar, 2012

\author{
Giovana LONGO-SILVA' \\ Risia Cristina Egito de MENEZES ${ }^{1}$ \\ Camila Alves Nogueira de SOUZA ${ }^{1}$ \\ Patrícia de Menezes MARINHO ${ }^{1}$ \\ Maysa Helena de Aguiar TOLONI ${ }^{2}$ \\ Maria Alice Araújo de OLIVEIRA ${ }^{1}$
}

\section{A B S T R A C T}

\section{Objective}

To investigate the frequency of consumption of obesogenic foods among adolescents and its association with sociodemographic, family, behavioral, and environmental variables.

\section{Methods}

Secondary data from the National School-Based Student Health Hurvey were analyzed from a representative sample of $9^{\text {th }}$ grade Brazilian students (high school). A self-administered questionnaire, organized into thematic blocks, was used. The dependent variables were the consumption of deep fried snacks, packaged snacks, sugar candies, and soft drinks; consumption frequency for the seven days preceding the study was analyzed. Bivariate analysis was carried out to determine the empirical relationship between the regular consumption of these foods ( $\geq 3$ days/week) with sociodemographic, family, behavioral, and school structural variables. $p$-value $<0.20$ was used as the criterion for initial inclusion in the multivariate logistic analysis, which was conducted using the "Enter" method, and the results were expressed as adjusted odds ratios with 95\% confidence interval and $p<0.05$ indicating a statistically significance.

\footnotetext{
1 Universidade Federal de Alagoas, Faculdade de Nutrição, Programa de Pós-Graduação em Nutrição. Campus AC Simões, Av. Lourival Melo Mota, s/n., Cidade Universitária, 57072-900, Maceió, AL, Brasil. Correspondência para/Correspondence to: G LONGO-SILVA. E-mail: <giovana_longo@yahoo.com.br>.

2 Universidade Federal de Lavras, Departamento de Nutrição, Setor Saúde Pública. Lavras, MG, Brasil.
} 


\section{Results}

Regular food consumption ranged from $27.17 \%$ to $65.96 \%$. The variables female gender, mobile phone ownership, Internet access at home, tobacco use, alcohol consumption, regular physical activity, eating while watching television or studying, watching television for at least 2 hours a day, and not willing to lose weight were associated in the final logistic models of all foods analyzed.

\section{Conclusion}

It was concluded that fried snacks, packaged snacks, sugar candies, and soft drinks are regularly consumed by adolescents and that such consumption was associated with the sociodemographic, family, behavioral, and school structural variables.

Keywords: Adolescent. Food consumption. Food habits. Industrialized foods.

\section{R E S U M O}

\section{Objetivo}

Analisar a frequência de consumo de alimentos obesogênicos entre adolescentes e sua associação com variáveis sociodemográficas, familiares, comportamentais e ambientais.

\section{Métodos}

Estudou-se dados secundários da Pesquisa Nacional de Saúde do Escolar, sendo a amostra representativa de alunos do $9^{\circ}$ ano do ensino fundamental de escolas brasileiras. Utilizou-se questionário autoaplicável organizado em blocos temáticos. Foram consideradas variáveis dependentes o consumo de salgados fritos, salgadinhos de pacote, guloseimas e refrigerantes, cuja frequência se referiu aos sete dias anteriores à pesquisa. Para associação do consumo regular dos alimentos ( $\geq 3$ dias/semana) às variáveis sociodemográficas, familiares, comportamentais e estruturais da escola, foi utilizada análise bivariada, adotando-se o critério de valor de $p<0,20$ para inclusão inicial na análise de regressão logística multivariada, a qual foi realizada pelo método "enter". Os resultados foram expressos por Odds Ratio ajustados com respectivos intervalos de confiança de 95\%, considerando associação estatisticamente significativa o valor de $p<0,05$.

\section{Resultados}

O consumo regular dos alimentos variou de $27,17 \%$ a $65,96 \%$. Sexo feminino, posse de telefone celular, acesso à Internet no domicílio, tabagismo, consumo de álcool, prática regular de atividade física, hábito de comer assistindo televisão ou estudando, assistir televisão no mínimo duas horas/dia e ausência de atitude visando perda de peso foram as variáveis que se associaram nos modelos logísticos finais de todos os alimentos.

\section{Conclusão}

Conclui-se que o consumo de salgados fritos, salgadinhos de pacote, guloseimas e refrigerantes é regular na dieta dos adolescentes, estando seu consumo associado a variáveis sociodemográficas, familiares, comportamentais e de estrutura escolar.

Palavras-chave: Adolescente. Consumo de alimentos. Hábitos alimentares. Alimentos industrializados.

\section{INTRODUCTION}

The term obesogenic refers to foods that promote gaining weight and that are not conducive to weight loss thus contributing to the obesity ${ }^{1}$. These foods include fast foods, massproduced foods that are prepared and served very quickly, usually in quick-service or self-service restaurants using pre-prepared ingredients. Some examples are hamburgers, French fries, pizza², and ultra-processed foods, which refer to those that are ready for consumption or heating with little or no preparation, such as a packaged snacks, cookies and crackers, sweet hard candies (including lollipop sticks), and soft drinks ${ }^{3}$.

The prevalence of obesity has increased dramatically in recent decades, affecting one in five adolescents in western countries ${ }^{4}$. Obesity in this population is of particular concern due to its negative psychological and health impacts with immediate and long term effects, including 
increased prevalence of cardiovascular risk factors, insulin resistance, type 2 diabetes, some types of cancers, low self-esteem, and reduced quality of life ${ }^{5}$.

Adolescent obesity can be attributed to endogenous causes related to specific behavioral characteristics defined by social bonding, social status, independence, and peer influence leading to sedentary behaviors, such as extensive television viewing and use of other electronic media, physical inactivity, eating away from home, and increased consumption of obesogenic foods ${ }^{6}$.

These foods are high in calories, total fat (saturated and trans fat), sugars, sodium, chemical food additives, and preservatives ${ }^{3}$, and are low in fiber, calcium, and iron. Their consumption is inversely associated with the consumption of fruits, vegetables, and milk?

Despite their harmful effects, convenience, affordability, palatability, and aggressive marketing are factors that contribute to keeping these food sin the daily diet. In Brazil, an analysis of the Pesquisas de Orçamentos Familiares (Household Budget Surveys) (1987-1988, 1995-1996, 2002-2003, and 2008-2009), showed that the caloric contribution of ultraprocessed foods (soft drinks, sugary drinks, encased meats, cookies and crackers, chocolates, sweets, and packaged snacks) to the total caloric value of the diet in the households increased linearly and significantly from $18.7 \%$ to $29.6 \%{ }^{8}$.

Thus, the analysis of the consumption frequency of these foods has been included into the adolescent health risk factor surveillance systems, which has been implemented following a recommendation by the World Health Organization (WHO). Therefore, inspired by systems of other countries, such as the Health Behavior in School-Aged Children ${ }^{9}$, Brazil conducted two national surveys in 2009 and 2012, which consisted of the Pesquisa Nacional de Saúde do Escolar (PeNSE, National SchoolBased Student Health Survey).

The present study aims, therefore, to investigate the frequency of consumption of obesogenic foods among adolescents and the sociodemographic, family, behavioral, and environmental factors associated with their regular consumption based on data from PeNSE-2012 .

\section{METHODS}

\section{Study population}

This study analyzed data from the second edition of the PeNSE, conducted by the Instituto Brasileiro de Geografia e Estatística (IBGE, Brazilian Institute of Geography and Statistics) in partnership with the Ministry of Health. That survey was conducted from April to September 2012, and the study population was composed of $9^{\text {th }}$ grade students (high school) attending public and private schools during the day.

This isa representative sample of the country, including the 5 macro-regions, the 26 state capitals, and the Federal District. Sample size was calculated considering $50 \%$ exposure prevalence, maximum error of 3\%, and $95 \%$ Confidence Interval $(95 \% \mathrm{Cl})$.

The sample of each geographic stratum was allocated in proportion to the number of schools, according to their administrative dependency (private or public schools). For each of these strata, a two-stage cluster sampling was used; in the first stage, the schools were selected, and in the second stage, the eligible classes in these schools were selected (high school $9^{\text {th }}$ graders). Thus, 132,123 students were invited to answer the questionnaire. However, at the time of data collection, only 110,873 were present, of which 1,651 declined to participate and 118 did not inform gender or age, and were thus excluded from the data base. The final sample was then composed of 109,104 students, representing a sample loss of $17.4 \%$.

The sample weight used in the data analysis was assigned to each student in the final sample, taking into account the sample loss 
mentioned above. Therefore, it should represent the target population, formed by high school $9^{\text {th }}$ graders who were regularly attending private and public schools during the day in the state capitals and the Federal District.

\section{Data collection}

Data were collected using mobile phones (smartphones); a self-report structured questionnaire was uploaded on the mobile devices. The questionnaire was organized into thematic blocks that included: sociodemographic characteristics, diet, body image, physical activity, tobacco use, alcohol and drug consumption, social protection network, oral health, sexual behavior, violence, and accidents. In addition to the information listed above, data related to work, hygiene, mental health, use of healthcare services, and prevalence of asthma were also collected.

\section{Dependent variables}

The dependent variables were the consumption of four foods, food groups, or prepared foods: deep fried snacks - such as French fries or coxinha de galinha (popular food in Brazil consisting of chopped or shredded chicken meat, covered in dough, molded into a shape resembling a chicken leg, battered and fried), deep fried kebab, pastel (a traditional deep fried Brazilian pastry filled with various sweet or savory ingredients), acarajé (a dish made from peeled beans formed into a ball and then deep-fried in palm oil), etc., - packaged snacks (including potato chips), sugar candies (sweets, hard candies, chocolates, chewing gum, and lollipop sticks), and soft drinks. Their consumption frequency for seven days preceding the study was analyzed.

Food consumption was expressed as absolute and relative frequencies, according to gender. For purposes of analysis, the variables were categorized as follows: consumption 3 or more days and 2 or fewer days in the seven days preceding the study ${ }^{10,11}$.

\section{Independent variables}

The independent variables were divided into sociodemographic: gender (male or female), age ( $\leq 14$ years or $>14$ years), currently employed (yes or no), household composition ( $\leq 4$ persons or $>4$ persons); home computer ownership (yes or no), Internet access at home (yes or no), mobile phone ownership (yes or no); family characteristics: maternal level of education $(<9$ years or $\geq 9$ years), living with the mother (yes or no), living with the father (yes or no); Behavioral: current use of tobacco (yes or no), current consumption of alcohol (yes or no), physical activity ( $\geq 3$ days or $<3$ days in the last 7 days), eating family meals (parents or guardians) ( $\geq 3$ days a week or $<3$ days a week), eating while watching television or studying ( $\geq 3$ days a week or $<3$ days a week), breakfast habits ( $\geq 3$ days a week or $<3$ days a week), time spent watching television ( $\geq 2$ hours, $<2$ hours), willingness to lose weight (yes or no); school structural: cafeteria (yes or no) and sports facilities (yes or no).

With regard to the variables current use of tobacco and current consumption of alcohol, use and consumption frequency for thirty days preceding the administration of the questionnaire was analyzed.

\section{Data analysis}

For the descriptive analysis, the independent variables were categorized estimating the total population proportion, according to the gender.

According to sociodemographic, family, behavioral, and school structural characteristics, regular food consumption ( $\geq 3$ days a week) was determined by simple frequency and confidence intervals. Bivariate analysis was used to investigate the factors associated with this eating habit. The chi-square test with Yates correction for dichotomous variables was used to compare the categorical variables of the groups, considering the $p$-value for statistical significance as $p<0.05$. 
To analyze the factors associated with regular food consumption, four multiple logistic regression models were used with the following outcomes: fried snacks, packaged snacks, sugar candies, and soft drinks. The variables with $p<0.20$ in the bivariate analysis were selected for initial inclusion in the multivariate logistic regression analysis. Odds ratios of regular food consumption (crude and adjusted for confounders) were calculated for each exposure variable.

Multiple logistic regression analysis was carried out using the "Enter" method. The results were expressed as adjusted ratios with $95 \% \mathrm{Cl}$, and variables with $p<0.05$ indicated a statistically significance.

Statistical analyses were performed using the Stata ${ }^{\circledR}$ software version 13.0 (Stata Corp, College Station, TX, USA) with the svy commands (survey commands), which are used for complex survey data analysis and include the required sampling weights, strata, and Primary Sampling Unit. The variables that were not answered (missing values) were excluded from the analysis.

\section{Ethical aspects}

Pesquisa Nacional de Saúde do Escolar was approved by the Comissão Nacional de Ética em Pesquisa (CONEP), appointed by the Ministry of Health (Protocol $n^{\circ} 16.805$ ), and authorized by the State and Municipal Departments of Education and the school board of the selected schools in each municipality.

\section{RES U L T S}

The sociodemographic characterization of the 109,104 adolescents studied showed a predominance of females (57.1\%) and age range of 11-19 years or more; the highest number of adolescents were aged below 14 years (68.4\%). Most students did not have a job and did not have a computer, mobile phone, and Internet access at home. With regard to the family aspects, about $10 \%$ and $30 \%$ of the students did not live with their mother and father, respectively (Table 1). As for the behavioral characteristics, less than half reported doing physical activities 3 or more days a week, with the higher percentage among the boys. It was also found that more than $75 \%$ of the students frequently ( 3 or more days a week) at eat least one of the meals with their parents or guardians, ate while watching television or studying, and did not eat the food offered at school.

In terms of the consumption of the food groups investigated in the present study (fried snacks, packaged snacks, sugar candies, and soft drinks), it was found that the proportion of students who regularly (three or more days a week) consumed these foods ranged between $27.17 \%$ and $65.96 \%$. The proportion of students who regularly consumed sugar candies $(60.9 \%)$ and soft drinks (56.2\%) was higher than that of those who regularly consumed packaged snacks (27.1\%) and fried snacks (35.6\%) (Table 2).

Analyzing these indicators of obesogenic food consumption, according to the sociodemographic variables for the students evaluated (Table 3), it was observed that the percentage of regular consumption of all foods analyzed was higher $(p<0.05)$ among those that had Internet access and owned a home computer and a mobile phone.

The analysis of the association between the variables related to behavioral characteristics, the consumption of obesogenic foods was higher among those who smoked, consumed alcohol, did physical activities three days or more days a week, watched television for more than two hours, and usually ate while watching TV or studying (Table 3). The same results were found in all final explanatory models after adjusting the variables (Table 4).

Table 4 shows the crude and adjusted odds ratios of the explanatory variables in relation to the consumption of fried snacks, packaged snacks, sugar candies, and soft drinks in the 
Table 1. Distribution of the population studied according to sociodemographic, family, behavioral, and school structural characteristics. Brazil, 2012.

1 of 2

\begin{tabular}{|c|c|c|c|c|c|c|}
\hline \multirow{2}{*}{ Variables } & \multicolumn{2}{|c|}{ Total } & \multicolumn{2}{|c|}{ Boys } & \multicolumn{2}{|c|}{ Girls } \\
\hline & $\mathrm{n}^{1}$ & $\% 2$ & $\mathrm{n}^{1}$ & $\% 2$ & $\mathrm{n}^{1}$ & $\% 2$ \\
\hline \multicolumn{7}{|c|}{ Sociodemographic } \\
\hline \multicolumn{7}{|l|}{ Age $(n=109,104)$} \\
\hline$\leq 14$ years & 73,343 & 68.4 & 32,388 & 63.7 & 40,955 & 72.8 \\
\hline$>14$ years & 35,761 & 31.6 & 19,627 & 36.3 & 16,134 & 27.2 \\
\hline \multicolumn{7}{|c|}{ Currently employed $(n=108,984)$} \\
\hline Yes & 14,318 & 13.1 & 91,01 & 17.4 & 5,217 & 9.2 \\
\hline No & 94,666 & 86.9 & 42,853 & 82.6 & 51,813 & 90.8 \\
\hline \multicolumn{7}{|c|}{ Household composition $(n=108,517)$} \\
\hline$\leq 4$ & 59,900 & 55.0 & 28,843 & 55.8 & 31,057 & 54.4 \\
\hline$>4$ & 48,617 & 45.0 & 22,859 & 44.2 & 25,758 & 45.6 \\
\hline \multicolumn{7}{|c|}{ Home computer ownership $(n=108,978)$} \\
\hline Yes & 74,350 & 66.0 & 36,624 & 68.5 & 37,726 & 63.7 \\
\hline No & 34,628 & 34.0 & 15,332 & 31.5 & 19,296 & 36.3 \\
\hline \multicolumn{7}{|c|}{ Internet access $(n=108,965)$} \\
\hline Yes & 69,233 & 60.4 & 33,984 & 62.6 & 35,249 & 58.4 \\
\hline No & 39,732 & 39.6 & 17,961 & 37.4 & 21,771 & 41.6 \\
\hline \multicolumn{7}{|c|}{ Mobile phone ownership $(n=108,984)$} \\
\hline Yes & 94,212 & 85.0 & 43,653 & 82.3 & 50,559 & 87.5 \\
\hline No & 14,772 & 15.0 & 83,05 & 17.7 & 6,467 & 12.5 \\
\hline \multicolumn{7}{|l|}{ Family } \\
\hline \multicolumn{7}{|c|}{ Maternal level of education $(n=90,577)$} \\
\hline$<9$ years & 33,322 & 42.3 & 14,768 & 40.0 & 18,554 & 44.4 \\
\hline$\geq 9$ years & 57,255 & 57.7 & 28,104 & 60.0 & 29,151 & 55.6 \\
\hline \multicolumn{7}{|c|}{ Living with the mother $(n=109,041)$} \\
\hline Yes & 97,016 & 90.6 & 46,206 & 90.6 & 50,810 & 90.7 \\
\hline No & 12,025 & 09.4 & 57,71 & 9.4 & 6,254 & 9.3 \\
\hline \multicolumn{7}{|c|}{ Living with the father $(n=108,942)$} \\
\hline Yes & 69,280 & 66.2 & 34,488 & 68.8 & 34,792 & 63.7 \\
\hline No & 39,662 & 33.8 & 17,450 & 31.2 & 22,212 & 36,3 \\
\hline \multicolumn{7}{|l|}{ Behavioral } \\
\hline \multicolumn{7}{|c|}{ Use of tobacco $(n=108,826)$} \\
\hline Yes & 57,48 & 5.1 & 29,37 & 5.1 & 28,11 & 5.0 \\
\hline No & 103,078 & 94.9 & 48,928 & 94.9 & 54,150 & 95.0 \\
\hline \multicolumn{7}{|c|}{ Consumption of alcohol $(n=108,668)$} \\
\hline Yes & 27,763 & 26.1 & 12,759 & 25.2 & 15,004 & 26.9 \\
\hline No & 80,905 & 73.9 & 39,001 & 74.8 & 41,904 & 73.1 \\
\hline \multicolumn{7}{|c|}{ Physical activity $(n=108,752)$} \\
\hline$\geq 3$ days a week & 46,788 & 41.4 & 27,625 & 51.2 & 19,163 & 32.5 \\
\hline$<3$ days a week & 61,964 & 58.6 & 24,195 & 48.8 & 37,769 & 67.5 \\
\hline \multicolumn{7}{|c|}{ Meals with parents or guardians $(n=108,888)$} \\
\hline$\geq 3$ days a week & 91,817 & 84.7 & 44928 & 86.8 & 46,889 & 82.8 \\
\hline$<3$ days a week & 17,071 & 15.3 & 6970 & 13.2 & 10,101 & 17.2 \\
\hline \multicolumn{7}{|c|}{ Eating while watching television or studying $(n=108,823)$} \\
\hline$\geq 3$ days a week & 83,322 & 77.4 & 41,104 & 80.1 & 42,218 & 75.0 \\
\hline$<3$ days a week & 25,501 & 22.6 & 10,755 & 19.9 & 14,746 & 25.0 \\
\hline Breakfast $(n=108$ & & & & & & \\
\hline$\geq 3$ days a week & 94,783 & 87.0 & 46,450 & 89.8 & 48,333 & 84.6 \\
\hline$<3$ days a week & 14,073 & 13.0 & 5,434 & 10.2 & 8,639 & 15.4 \\
\hline
\end{tabular}


Table 1. Distribution of the population studied according to sociodemographic, family, behavioral, and school structural characteristics. Brazil, 2012.

2 of 2

\begin{tabular}{|c|c|c|c|c|c|c|}
\hline \multirow{2}{*}{ Variables } & \multicolumn{2}{|c|}{ Total } & \multicolumn{2}{|c|}{ Boys } & \multicolumn{2}{|c|}{ Girls } \\
\hline & $\mathrm{n}^{1}$ & $\%^{2}$ & $n^{1}$ & $\%^{2}$ & $n^{1}$ & $\%^{2}$ \\
\hline \multicolumn{7}{|l|}{ Behavioral } \\
\hline \multicolumn{7}{|c|}{ Time spent watching television $(n=108,792)$} \\
\hline$\geq 2$ hours a day & 67,672 & 63.6 & 30,821 & 60.5 & 36,851 & 66.6 \\
\hline$<2$ hours a day & 41,120 & 36.4 & 21,024 & 39.5 & 20,096 & 33.4 \\
\hline \multicolumn{7}{|c|}{ Willingness to lose weight $(n=108.447)$} \\
\hline Yes & 29,144 & 26.3 & 11,119 & 21.1 & 18,025 & 31.1 \\
\hline No & 79,303 & 73.7 & 40,493 & 78.9 & 38,810 & 68.9 \\
\hline \multicolumn{7}{|c|}{ School food consumption $(n=108,854)$} \\
\hline$\geq 3$ days a week & 22,753 & 20.9 & 12,079 & 23.3 & 10,674 & 18.7 \\
\hline$<3$ days a week & 86,101 & 79.1 & 39,802 & 76.7 & 46,299 & 81.3 \\
\hline \multicolumn{7}{|c|}{ School structural } \\
\hline \multicolumn{7}{|c|}{ Cafeteria $(N=108,837)$} \\
\hline Yes & 55,645 & 51.1 & 26,961 & 50.3 & 28,684 & 47.6 \\
\hline No & 53,192 & 48.9 & 24,937 & 49.7 & 28,255 & 52.4 \\
\hline \multicolumn{7}{|c|}{ Sports facilities $(N=108,795)$} \\
\hline Yes & 88,534 & 79.4 & 42,609 & 81.0 & 45,925 & 77.9 \\
\hline No & 20,261 & 20.6 & 9,270 & 19.0 & 10,991 & 22.1 \\
\hline
\end{tabular}

Note: ${ }^{1}$ Number of individuals in the unweighted sample; ${ }^{2}$ Prevalence calculated as weighted confidence interval due to the sampling design.

Table 2. Distribution of the population studied in terms of the frequency of consumption of obsegenic foods according to gender. Brazil, 2012.

\begin{tabular}{|c|c|c|c|c|c|c|c|c|c|}
\hline \multirow{3}{*}{ Foods } & \multicolumn{9}{|c|}{ Weekly consumption frequency (\%) } \\
\hline & \multirow{2}{*}{$\begin{array}{c}\text { Total } \\
n^{1}\end{array}$} & \multicolumn{2}{|c|}{4 days } & \multicolumn{2}{|c|}{5 days } & \multicolumn{2}{|c|}{6 days } & \multicolumn{2}{|c|}{7 days } \\
\hline & & $\mathrm{n}^{1}$ & $\%^{2}$ & $n^{1}$ & $\%^{2}$ & $n^{1}$ & $\%^{2}$ & $n^{1}$ & $\%^{2}$ \\
\hline Fried snacks & 108,889 & 7,482 & 6.8 & 5,326 & 4.7 & 2,773 & 2.7 & 8,888 & 8.4 \\
\hline Boys & 51,904 & 3,466 & 6.7 & 2,383 & 4.5 & 1,206 & 2.4 & 3,703 & 7.3 \\
\hline Girls & 56,985 & 4,016 & 7.0 & 2,943 & 5.0 & 1,567 & 2.9 & 5,185 & 9.4 \\
\hline Packaged snacks & 108,804 & 5,407 & 5.3 & 4,024 & 4.1 & 2,696 & 2.8 & 5,942 & 6.1 \\
\hline Boys & 51,863 & 2,506 & 5.2 & 1,649 & 3.6 & 1,019 & 2.2 & 2,353 & 5.1 \\
\hline Girls & 56,941 & 2,900 & 5.5 & 2,375 & 4.6 & 1,677 & 3.3 & 3,589 & 7.0 \\
\hline Sugar candies & 108,815 & 9,149 & 8.3 & 8,236 & 7.4 & 6,378 & 5.9 & 29,813 & 27.9 \\
\hline Boys & 51,867 & 4,467 & 8.5 & 3,905 & 7.4 & 2,587 & 5.0 & 10,952 & 21.4 \\
\hline Girls & 56,948 & 4,682 & 8.1 & 4,331 & 7.5 & 3,791 & 6.7 & 18,861 & 34.0 \\
\hline Soft drinks & 108,858 & 10,552 & 9.5 & 8,867 & 7.9 & 6,361 & 5.9 & 20,373 & 19.4 \\
\hline Boys & 51,874 & 5,403 & 10.3 & 4,448 & 8.4 & 2,900 & 5.7 & 9,760 & 19.5 \\
\hline Girls & 56,984 & 5,149 & 8.7 & 4,419 & 7.5 & 3,461 & 6.1 & 10,613 & 19.3 \\
\hline
\end{tabular}

Note: ${ }^{1}$ Number of individuals in the unweighted sample; ${ }^{2}$ Prevalence calculated as weighted confidence interval due to the sampling design.

multiple logistic regression analysis. Among the factors that remained in the final model as factors associated with the consumption of fried snacks and packaged snacks, the variables female gender, currently employed, Internet access, mobile phone ownership, consumption of alcohol, use of tobacco, and spending more than 2 hours a day watching television are some of the conditions that indicated greater probability of regular consumption of these foods. 
Table 3. Regular consumption ( $\geq 3$ days a week) of obesogenic foods among adolescents, prevalence, and respective $95 \%$ confidence intervals, according to sociodemographic, family, behavioral, and school structural characteristics. Brazil, 2012.

\begin{tabular}{|c|c|c|c|c|c|}
\hline \multirow{2}{*}{ Variables } & \multicolumn{5}{|c|}{ Fried snacks } \\
\hline & $n^{1}$ & $\mathrm{n}^{1}$ & $\%^{2}$ & $(95 \% \mathrm{Cl})^{2}$ & $p^{*}$ \\
\hline \multicolumn{6}{|l|}{ Sociodemographic } \\
\hline Gender & 108,889 & & & & \\
\hline Male & & 17,615 & 34.6 & $32.87-36.33$ & 0.0069 \\
\hline Female & & 20,765 & 36.7 & $35.16-38.32$ & \\
\hline Age & 108,889 & & & & \\
\hline$\leq 14$ years & & 25,366 & 35.3 & $33.76-36.83$ & 0.0223 \\
\hline$>14$ years & & 13,014 & 36.6 & $35-38.24$ & \\
\hline Currently employed & 108,845 & & & & \\
\hline Yes & & 5,936 & 41.9 & $39.22-44.57$ & $<0.0001$ \\
\hline No & & 32,430 & 34.8 & $33.41-36.15$ & \\
\hline Household composition & 108,387 & & & & \\
\hline$\leq 4$ persons & & 21,494 & 36.2 & $34.8-37.63$ & 0.0718 \\
\hline$>4$ persons & & 16,738 & 35.1 & $33.46-36.86$ & \\
\hline Home computer ownership & 108,847 & & & & \\
\hline Yes & & 28,090 & 38.5 & $37.37-39.61$ & $<0.0001$ \\
\hline No & & 10,269 & 30.3 & $27.95-32.71$ & \\
\hline Internet access & 108,831 & & & & \\
\hline Yes & & 26,798 & 39.6 & $38.49-40.68$ & $<0.0001$ \\
\hline No & & 11,557 & 29.8 & $27.8-31.78$ & \\
\hline Mobile phone ownership & 108,848 & & & & \\
\hline Yes & & 34,308 & 37.0 & $35.6-38.38$ & $<0.0001$ \\
\hline No & & 4,058 & 28.4 & $26.6-30.32$ & \\
\hline \multicolumn{6}{|l|}{ Family } \\
\hline Maternal level of education & 90,456 & & & & \\
\hline$<9$ years & & 10,661 & 32.7 & $30.63-34.8$ & $<0.0001$ \\
\hline$\geq 9$ years & & 21,701 & 38.7 & $37-3740$ & \\
\hline Living with the mother & 108,850 & & & & \\
\hline Yes & & 34,090 & 35.6 & $34.14-37.16$ & 0.3710 \\
\hline No & & 4,277 & 36.3 & $34.96-37.7$ & \\
\hline Living with the father & 108,761 & & & & \\
\hline Yes & & 23,812 & 34.8 & $33.33-36.41$ & $<0.0001$ \\
\hline No & & 14,517 & 37.3 & $35.96-38.69$ & \\
\hline \multicolumn{6}{|l|}{ Behavioral } \\
\hline Use of tobacco & 108,732 & & & & \\
\hline Yes & & 2,673 & 45.2 & $42.9-47.43$ & $<0.0001$ \\
\hline No & & 35,639 & 35.2 & $33.83-36.58$ & \\
\hline Consumption of alcohol & 108,574 & & & & \\
\hline Yes & & 12,400 & 44.7 & $42.71-46.71$ & $<0.0001$ \\
\hline No & & 25,852 & 32.5 & $31.19-33.88$ & \\
\hline Physical activity & 108,666 & & & & \\
\hline$\geq 3$ days a week & & 17,543 & 38.4 & $36.61-40.12$ & $<0.0001$ \\
\hline$<3$ days a week & & 20,747 & 33.8 & $32.59-35.1$ & \\
\hline Meals with parents or guardians & 108,792 & & & & \\
\hline$\geq 3$ days a week & & 31,796 & 35.2 & $33.83-36.63$ & $<0.0001$ \\
\hline$<3$ days a week & & 6,543 & 38.4 & $36.5-40.27$ & \\
\hline Eating while watching television or studying & 108,734 & & & & \\
\hline$\geq 3$ days a week & & 30,069 & 36.6 & $35.09-38.14$ & $<0.0001$ \\
\hline$<3$ days a week & & 8,243 & 32.6 & $30.85-34.33$ & \\
\hline
\end{tabular}


Table 3. Regular consumption ( $\geq 3$ days a week) of obesogenic foods among adolescents, prevalence, and respective $95 \%$ confidence intervals, according to sociodemographic, family, behavioral, and school structural characteristics. Brazil, 2012.

\begin{tabular}{|c|c|c|c|c|c|}
\hline \multirow{2}{*}{ Variables } & \multicolumn{5}{|c|}{ Fried snacks } \\
\hline & $n^{1}$ & $n^{1}$ & $\%^{2}$ & $(95 \% \mathrm{Cl})^{2}$ & $p^{*}$ \\
\hline \multicolumn{6}{|l|}{ Behavioral } \\
\hline Breakfast & 108,761 & & & & \\
\hline$\geq 3$ days a week & & 33,041 & 35.4 & $33.89-37.03$ & 0.0056 \\
\hline$<3$ days a week & & 5,287 & 37.4 & $36.26-38.6$ & \\
\hline Time spent watching television & 108,698 & & & & \\
\hline$\geq 2$ hours a day & & 25,849 & 38.9 & $37.45-40.43$ & $<0.0001$ \\
\hline$<2$ hours a day & & 12,452 & 30.0 & $28.52-31.53$ & \\
\hline Willingness to lose weight & 108,355 & & & & \\
\hline Yes & & 9,168 & 31.8 & $30.77-32.88$ & $<0.0001$ \\
\hline No & & 29,017 & 37.1 & $35.37-38.81$ & \\
\hline School food consumption & 108,766 & & & & \\
\hline$\geq 3$ days a week & & 7,343 & 33.2 & $30.68-35.88$ & 0.0359 \\
\hline$<3$ days a week & & 30,991 & 36.4 & $34.76-37.98$ & \\
\hline Cafeteria & 108,622 & & & & \\
\hline Yes & & 20,698 & 37.7 & $36.17-39.18$ & 0.0010 \\
\hline No & & 17,572 & 33.8 & $31.72-35.98$ & \\
\hline Sports facilities & 108,580 & & & & \\
\hline Yes & & 31,723 & 36.6 & $35.14-38.15$ & 0.0002 \\
\hline No & & 6,528 & 32.1 & $30.07-34.24$ & \\
\hline \multirow{2}{*}{ Variables } & \multicolumn{5}{|c|}{ Packaged snacks } \\
\hline & $\mathrm{n}^{1}$ & $\mathrm{n}^{1}$ & $\%^{2}$ & $(95 \% \mathrm{Cl})^{2}$ & $p^{*}$ \\
\hline \multicolumn{6}{|l|}{ Sociodemographic } \\
\hline Gender & 108,804 & & & & \\
\hline Male & & 11,771 & 24.7 & $23.52-25.92$ & $<0.0001$ \\
\hline Female & & 15,405 & 29.4 & $28.3-30.61$ & \\
\hline Age & 108,804 & & & & \\
\hline$\leq 14$ years & & 17,769 & 26.8 & $25.48-28.1$ & 0.0934 \\
\hline$>14$ years & & 9,407 & 28.1 & $26.9-29.25$ & \\
\hline Currently employed & 108,762 & & & & \\
\hline Yes & & 4,116 & 30.7 & $29.11-32.36$ & $<0.0001$ \\
\hline No & & 23,048 & 26.6 & $25.7-27.61$ & \\
\hline Household composition & 108,305 & & & & \\
\hline$\leq 4$ persons & & 14,878 & 27.0 & $26.21-27.88$ & 0.4468 \\
\hline$>4$ persons & & 12,160 & 27.4 & $26.06-28.67$ & \\
\hline Home computer ownership & 108,764 & & & & \\
\hline Yes & & 19,390 & 28.4 & $27.74-29.04$ & 0.0001 \\
\hline No & & 7,771 & 24.8 & $22.95-26.78$ & \\
\hline Internet access & 108,752 & & & & \\
\hline Yes & & 18,455 & 29.0 & $28.36-29.73$ & $<0.0001$ \\
\hline No & & 8,700 & 24.3 & $22.8-25.91$ & \\
\hline Mobile phone ownership & 108,772 & & & & \\
\hline Yes & & 24,214 & 28.0 & $26.98-29.09$ & $<0.0001$ \\
\hline No & & 2,952 & 22.4 & $21.1-23.68$ & \\
\hline \multicolumn{6}{|l|}{ Family } \\
\hline Maternal level of education & 90,373 & & & & \\
\hline$<9$ years & & 8,186 & 26.7 & $24.67-28.81$ & 0.1424 \\
\hline$\geq 9$ years & & 14,656 & 28.1 & $27.48-28.65$ & \\
\hline
\end{tabular}


Table 3. Regular consumption ( $\geq 3$ days a week) of obesogenic foods among adolescents, prevalence, and respective $95 \%$ confidence intervals, according to sociodemographic, family, behavioral, and school structural characteristics. Brazil, 2012.

\begin{tabular}{|c|c|c|c|c|c|}
\hline \multirow{2}{*}{ Variables } & \multicolumn{5}{|c|}{ Packaged snacks } \\
\hline & $n^{1}$ & $n^{1}$ & $\%^{2}$ & $(95 \% \mathrm{Cl})^{2}$ & $p^{*}$ \\
\hline \multicolumn{6}{|l|}{ Family } \\
\hline Living with the mother & 108,766 & & & & \\
\hline Yes & & 24,123 & 27.2 & $26.15-28.19$ & 0.7142 \\
\hline No & & 3,043 & 27.4 & $26.12-28.63$ & \\
\hline Living with the father & 108,680 & & & & \\
\hline Yes & & 16,907 & 26.6 & $25.5-27.75$ & $<0.0001$ \\
\hline No & & 10,233 & 28.3 & $27.36-29.22$ & \\
\hline \multicolumn{6}{|l|}{ Behavioral } \\
\hline Use of tobacco & 108,673 & & & & \\
\hline Yes & & 2,084 & 39.2 & $36.56-41.83$ & $<0.0001$ \\
\hline No & & 25,057 & 26.5 & $25.67-27.43$ & \\
\hline Consumption of alcohol & 108,515 & & & & \\
\hline Yes & & 9,042 & 34.8 & $32.87-36.86$ & $<0.0001$ \\
\hline No & & 18,047 & 24.5 & $23.67-25.27$ & \\
\hline Physical activity & 108,612 & & & & \\
\hline$\geq 3$ days a week & & 12,564 & 29.3 & $27.77-30.93$ & $<0.0001$ \\
\hline$<3$ days a week & & 14,560 & 25.7 & $24.96-26.39$ & \\
\hline Meals with parents or guardians & 108,733 & & & & \\
\hline$\geq 3$ days a week & & 22,605 & 26.9 & $25.82-27.92$ & 0.0033 \\
\hline$<3$ days a week & & 4,553 & 28.9 & $27.55-30.35$ & \\
\hline Eating while watching television or studying & 108,673 & & & & \\
\hline$\geq 3$ days a week & & 21,553 & 28.0 & $27.01-29.05$ & $<0.0001$ \\
\hline$<3$ days a week & & 5,581 & 24.2 & $23.1-25.38$ & \\
\hline Breakfast & 108,706 & & & & \\
\hline$\geq 3$ days a week & & 23,472 & 27.1 & $26.07-28.14$ & 0.0649 \\
\hline$<3$ days a week & & 3,676 & 27.7 & $26.8-28.63$ & \\
\hline Time spent watching television & 108,639 & & & & \\
\hline$\geq 2$ hours a day & & 18,907 & 30.3 & $29.16-31.53$ & $<0.0001$ \\
\hline$<2$ hours a day & & 8,226 & 21.6 & $20.9-22.41$ & \\
\hline Willingness to lose weight & 108,294 & & & & \\
\hline Yes & & 6,210 & 23.3 & $22.19-24.43$ & $<0.0001$ \\
\hline No & & 20,812 & 28.5 & $27.38-29.67$ & \\
\hline School food consumption & 108,703 & & & & \\
\hline$\geq 3$ days a week & & 5,566 & 26.8 & $25.61-28.01$ & 0.3037 \\
\hline$<3$ days a week & & 21,582 & 27.3 & $26.26-28.32$ & \\
\hline \multicolumn{6}{|l|}{ School structural } \\
\hline Cafeteria & 108,537 & & & & \\
\hline Yes & & 13,657 & 27.1 & $25.21-29.13$ & 0.9359 \\
\hline No & & 13,456 & 27.2 & $25.74-28.8$ & \\
\hline Sports facilities & 108,495 & & & & \\
\hline Yes & & 22,085 & 27.7 & $26.74-28.62$ & 0.0455 \\
\hline No & & 5,017 & 25.3 & $23.24-27.55$ & \\
\hline \multirow{2}{*}{ Variables } & \multicolumn{5}{|c|}{ Sugar candies } \\
\hline & $n^{1}$ & $\mathrm{n}^{1}$ & $\%^{2}$ & $(95 \% \mathrm{Cl})^{2}$ & $p^{*}$ \\
\hline \multicolumn{6}{|l|}{ Sociodemographic } \\
\hline Gender & 108,815 & & & & \\
\hline Male & & 28,291 & 54.7 & $51.7-57.58$ & $<0.0001$ \\
\hline Female & & 37,675 & 66.7 & $64.17-69.07$ & \\
\hline
\end{tabular}


Table 3. Regular consumption ( $\geq 3$ days a week) of obesogenic foods among adolescents, prevalence, and respective $95 \%$ confidence intervals, according to sociodemographic, family, behavioral, and school structural characteristics. Brazil, 2012.

4 of 6

\begin{tabular}{|c|c|c|c|c|c|}
\hline \multirow{2}{*}{ Variables } & \multicolumn{5}{|c|}{ Sugar candies } \\
\hline & $n^{1}$ & $\mathrm{n}^{1}$ & $\%^{2}$ & $(95 \% \mathrm{Cl})^{2}$ & $p^{*}$ \\
\hline \multicolumn{6}{|l|}{ Sociodemographic } \\
\hline Age & 108,815 & & & & \\
\hline$\leq 14$ years & & 45,824 & 63.0 & $60.7-65.19$ & $<0.0001$ \\
\hline$>14$ years & & 20,142 & 56.5 & $54.48-58.45$ & \\
\hline Currently employed & 108,773 & & & & \\
\hline Yes & & 8,711 & 61.5 & $60.11-62.96$ & 0.5608 \\
\hline No & & 57,237 & 60.8 & $58.11-63.52$ & \\
\hline Household composition & 108,317 & & & & \\
\hline$\leq 4$ persons & & 36,722 & 61.7 & $58.6-64.69$ & 0.1117 \\
\hline$>4$ persons & & 28,994 & 60.1 & $58.05-62.17$ & \\
\hline Home computer ownership & 108,775 & & & & \\
\hline Yes & & 46,569 & 63.2 & $60.02-66.34$ & $<0.0001$ \\
\hline No & & 19,376 & 56.4 & $54.38-58.46$ & \\
\hline Internet access & 108,763 & & & & \\
\hline Yes & & 43,730 & 63.7 & $60.4-66.93$ & $<0.0001$ \\
\hline No & & 22,210 & 56.7 & $54.5-58.8$ & \\
\hline Mobile phone ownership & 108,780 & & & & \\
\hline Yes & & 58,138 & 62.3 & $59.61-64.84$ & $<0.0001$ \\
\hline No & & 7,810 & 53.3 & $51.96-54.66$ & \\
\hline \multicolumn{6}{|l|}{ Family } \\
\hline Maternal level of education & 90,384 & & & & \\
\hline$<9$ years & & 19,210 & 58.0 & $55.57-60.37$ & $<0.0001$ \\
\hline$\geq 9$ years & & 35,728 & 63.2 & $60.26-66$ & \\
\hline Living with the mother & 108,775 & & & & \\
\hline Yes & & 58,771 & 61.0 & $58.35-63.52$ & 0.6294 \\
\hline No & & 7,175 & 60.5 & $59.16-61.9$ & \\
\hline Living with the father & 108,689 & & & & \\
\hline Yes & & 41,616 & 60.6 & $57.83-63.26$ & 0.0384 \\
\hline No & & 24,294 & 61.6 & $59.6-63.67$ & \\
\hline \multicolumn{6}{|l|}{ Behavioral } \\
\hline Use of tobacco & 108.680 & & & & $<0.0001$ \\
\hline Yes & & 3,918 & 69.0 & $66.47-71.32$ & \\
\hline No & & 61,973 & 60.5 & $58.1-62.86$ & \\
\hline Consumption of alcohol & 108,531 & & & & $<0.0001$ \\
\hline Yes & & 18,969 & 68.7 & $66.71-70.63$ & \\
\hline No & & 46,841 & 58.2 & $55.61-60.77$ & \\
\hline Physical activity & 108,621 & & & & $<0.0001$ \\
\hline$\geq 3$ days a week & & 29,267 & 63.5 & $60.92-65.99$ & \\
\hline$<3$ days a week & & 36,603 & 59.2 & $56.72-61.55$ & \\
\hline Meals with parents or guardians & 108,744 & & & & $<0.0001$ \\
\hline$\geq 3$ days a week & & 55,077 & 60.3 & $57.81-62.8$ & \\
\hline$<3$ days a week & & 10,849 & 64.3 & $61.66-66.9$ & \\
\hline Eating while watching television or studying & 108,683 & & & & \\
\hline$\geq 3$ days a week & & 50,939 & 61.4 & $59.04-63.74$ & 0.0001 \\
\hline$<3$ days a week & & 14,953 & 59.2 & $56.25-62.15$ & \\
\hline Breakfast & 108,712 & & & & $<0.0001$ \\
\hline$\geq 3$ days a week & & 56,905 & 60.4 & $57.83-62.93$ & \\
\hline$<3$ days a week & & 9,008 & 64.5 & $62.26-66.68$ & \\
\hline
\end{tabular}


Table 3. Regular consumption ( $\geq 3$ days a week) of obesogenic foods among adolescents, prevalence, and respective $95 \%$ confidence intervals, according to sociodemographic, family, behavioral, and school structural characteristics. Brazil, 2012.

\begin{tabular}{|c|c|c|c|c|c|}
\hline \multirow{2}{*}{ Variables } & \multicolumn{5}{|c|}{ Sugar candies } \\
\hline & $\mathrm{n}^{1}$ & $n^{1}$ & $\%^{2}$ & $(95 \% \mathrm{Cl})^{2}$ & $p^{*}$ \\
\hline \multicolumn{6}{|l|}{ Behavioral } \\
\hline Time spent watching television & 108,655 & & & & \\
\hline$\geq 2$ hours a day & & 43,905 & 65.2 & $62.27-68.04$ & $<0.0001$ \\
\hline$<2$ hours a day & & 21,978 & 53.5 & $51.47-55.49$ & \\
\hline Willingness to lose weight & 108,309 & & & & \\
\hline Yes & & 17,105 & 59.1 & $56.13-61.95$ & $<0.0001$ \\
\hline No & & 48,604 & 61.6 & $59.3-63.94$ & \\
\hline School food consumption & 108,716 & & & & \\
\hline$\geq 3$ days a week & & 13,131 & 57.7 & $56.19-59.26$ & $<0.0001$ \\
\hline$<3$ days a week & & 52,781 & 61.8 & $58.92-64.56$ & \\
\hline \multicolumn{6}{|l|}{ School structural } \\
\hline Cafeteria & 108,548 & & & & \\
\hline Yes & & 34,423 & 62.7 & 60.11 .65 .27 & 0.0001 \\
\hline No & & 31,357 & 59.2 & 57.34 .60 .97 & \\
\hline Sports facilities & 108,506 & & & & \\
\hline Yes & & 54,115 & 61.8 & $59.04-64.46$ & 0.1054 \\
\hline No & & 11,639 & 57.6 & $53.3-61.69$ & \\
\hline \multirow{2}{*}{ Variables } & \multicolumn{5}{|c|}{ Soft drinks } \\
\hline & $n^{1}$ & $n^{1}$ & $\%^{2}$ & $(95 \% \mathrm{Cl})^{2}$ & $p^{*}$ \\
\hline \multicolumn{6}{|l|}{ Sociodemographic } \\
\hline Gender & 108,858 & & & & \\
\hline Male & & 30,004 & 58.1 & $56.57-59.65$ & $<0.0001$ \\
\hline Female & & 30,986 & 54.2 & $51.71-56.58$ & \\
\hline Age & 108,858 & & & & \\
\hline$\leq 14$ years & & 40,537 & 55.5 & $54.32-56.62$ & 0.2258 \\
\hline$>14$ years & & 20,453 & 57.3 & $53.44-61.06$ & \\
\hline Currently employed & 108,814 & & & & \\
\hline Yes & & 8,995 & 62.8 & $61.33-64.35$ & $<0.0001$ \\
\hline No & & 51,973 & 55.0 & $52.92-57.12$ & \\
\hline Household composition & 108,359 & & & & \\
\hline$\leq 4$ persons & & 34,098 & 57.5 & $56.08-58.92$ & 0.0001 \\
\hline$>4$ persons & & 26,658 & 54.4 & $51.79-56.98$ & \\
\hline Home computer ownership & 108,814 & & & & \\
\hline Yes & & 44,195 & 60.4 & $59.35-61.49$ & $<0.0001$ \\
\hline No & & 16,772 & 47.6 & $44.02-51.12$ & \\
\hline Internet access & 108,800 & & & & \\
\hline Yes & & 41,650 & 61.4 & $60.37-62.31$ & $<0.0001$ \\
\hline No & & 19,313 & 48.0 & $44.75-51.24$ & \\
\hline Mobile phone ownership & 108,817 & & & & \\
\hline Yes & & 54,031 & 57.5 & $55.73-59.26$ & $<0.0001$ \\
\hline No & & 6,940 & 47.7 & $44.23-51.26$ & \\
\hline \multicolumn{6}{|l|}{ Family } \\
\hline Maternal level of education & 90,412 & & & & \\
\hline$<9$ years & & 17,211 & 51.7 & $48.97-54.4$ & $<0.0001$ \\
\hline$\geq 9$ years & & 33,459 & 59.2 & $57.52-60.87$ & \\
\hline Living with the mother & 108,817 & & & & \\
\hline Yes & & 54,192 & 56.0 & $54.04-58.04$ & 0.9659 \\
\hline No & & 6,778 & 56.1 & $54.14-57.98$ & \\
\hline
\end{tabular}


Table 3. Regular consumption ( $\geq 3$ days a week) of obesogenic foods among adolescents, prevalence, and respective $95 \%$ confidence intervals, according to sociodemographic, family, behavioral, and school structural characteristics. Brazil, 2012.

6 of 6

\begin{tabular}{|c|c|c|c|c|c|}
\hline \multirow{2}{*}{ Variables } & \multicolumn{5}{|c|}{ Soft drinks } \\
\hline & $n^{1}$ & $n^{1}$ & $\%^{2}$ & $(95 \% \mathrm{Cl})^{2}$ & $p^{*}$ \\
\hline \multicolumn{6}{|l|}{ Family } \\
\hline Living with the father & 108,728 & & & & \\
\hline Yes & & 38,428 & 55.6 & $53.6-57.62$ & 0.1371 \\
\hline No & & 22,502 & 56.9 & $54.62-59.22$ & \\
\hline \multicolumn{6}{|l|}{ Behavioral } \\
\hline Use of tobacco & 108,721 & & & & \\
\hline Yes & & 3,875 & 65.6 & $62.12-68.99$ & $<0.0001$ \\
\hline No & & 57,043 & 55.6 & $53.55-57.54$ & \\
\hline Consumption of alcohol & 108,570 & & & & \\
\hline Yes & & 18,355 & 65.5 & $63.72-67.23$ & $<0.0001$ \\
\hline No & & 42,465 & 52.7 & $50.46-54.99$ & \\
\hline Physical activity & 108,655 & & & & \\
\hline$\geq 3$ days a week & & 27,269 & 58.8 & $56.57-61.05$ & $<0.0001$ \\
\hline$<3$ days a week & & 33,611 & 54.1 & $52.33-55.86$ & \\
\hline Meals with parents or guardians & 108,794 & & & & \\
\hline$\geq 3$ days a week & & 51,237 & 55.9 & $53.93-57.83$ & 0.0318 \\
\hline$<3$ days a week & & 9,724 & 57.0 & $54.76-59.14$ & \\
\hline Eating while watching television or studying & 108.729 & & & & \\
\hline$\geq 3$ days a week & & 47,507 & 57.1 & $54.94-59.19$ & $<0.0001$ \\
\hline$<3$ days a week & & 13,410 & 52.5 & $50.55-54.44$ & \\
\hline Breakfast & 108,759 & & & & \\
\hline$\geq 3$ days a week & & 52,655 & 55.7 & $53.76-57.63$ & $<0.0001$ \\
\hline$<3$ days a week & & 8,286 & 58.4 & $56.22-60.55$ & \\
\hline Time spent watching television & 108,694 & & & & \\
\hline$\geq 2$ hours a day & & 40,195 & 59.4 & $57.57-61.2$ & $<0.0001$ \\
\hline$<2$ hours a day & & 20,698 & 50.2 & $47.96-52.44$ & \\
\hline Willingness to lose weight & 108,348 & & & & \\
\hline Yes & & 15,237 & 52.2 & $50.9-53.54$ & $<0.0001$ \\
\hline No & & 45,476 & 57.4 & $55.1-59.71$ & \\
\hline School food consumption & 108,760 & & & & \\
\hline$\geq 3$ days a week & & 12,165 & 52.8 & $49.83-55.66$ & $<0.0001$ \\
\hline$<3$ days a week & & 48,774 & 56.9 & $55.08-58.75$ & \\
\hline \multicolumn{6}{|l|}{ School structural } \\
\hline Cafeteria & 108,591 & & & & \\
\hline Yes & & 32,108 & 57.8 & $50.03-58.62$ & 0.1195 \\
\hline No & & 28,728 & 54.4 & $56.82-58.82$ & \\
\hline Sports facilities & 108,549 & & & & \\
\hline Yes & & 50,383 & 57.5 & $56.19-58.83$ & 0.0025 \\
\hline No & & 10,432 & 50.4 & $45.19-55.67$ & \\
\hline
\end{tabular}

Note: " $p$-value - Pearson's chi-square test; ${ }^{1}$ Number of individuals in the unweighted sample; ${ }^{2}$ Prevalence and $95 \%$ confidence intervals $(95 \% \mathrm{Cl})$, calculated as weighted confidence interval due to the sampling design.

95\% Cl : 95\% Confidence Interval.

It is worth mentioning that the existence of cafeteria at school was not associated with the consumption of packaged snacks and soft drinks ( $p>0.05)$ in the crude analysis. After adjustment of the model to the other independent variables in the final model, the existence of cafeteria at school was associated only withsugar candies (OR 1.8; 95\% Cl 1.05 to 1.12) (Table 4). 
Table 4. Multiple logistic regression analysis for the regular consumption of obesogenic foods among adolescents, according to sociodemographic, family, behavioral, and school structural characteristics. Brazil, 2012.

\begin{tabular}{|c|c|c|c|c|c|c|}
\hline \multirow{3}{*}{ Variables } & \multicolumn{6}{|c|}{ Fried snacks } \\
\hline & \multicolumn{3}{|c|}{ Crude odds Ratio } & \multicolumn{3}{|c|}{ Adjusted odds Ratio } \\
\hline & $\mathrm{OR}^{1}$ & $(95 \% \mathrm{Cl})^{2}$ & $p$ & $\mathrm{OR}^{1}$ & $(95 \% \mathrm{Cl})^{2}$ & $p$ \\
\hline \multicolumn{7}{|c|}{ Sociodemographic } \\
\hline \multicolumn{7}{|l|}{ Gender } \\
\hline Male & 1 & - & - & - & - & - \\
\hline Female & 1.09 & $1.02-1.17$ & 0.007 & 0.007 & $1.11-1.29$ & $<0.0001$ \\
\hline \multicolumn{7}{|l|}{ Age } \\
\hline$\leq 14$ years & 1 & - & - & - & - & - \\
\hline$>14$ years & 1.05 & $1.00-1.11$ & 0.022 & 0.022 & $1.00-1.13$ & 0.027 \\
\hline \multicolumn{7}{|c|}{ Currently employed } \\
\hline Yes & 1.35 & $1.25-1.35$ & $<0.0001$ & $<0.0001$ & $1.18-1.43$ & $<0.0001$ \\
\hline No & 1 & - & - & - & - & - \\
\hline \multicolumn{7}{|c|}{ Household composition } \\
\hline$\leq 4$ persons & 1 & - & - & - & - & - \\
\hline$>4$ persons & 0.95 & $0.90-1.00$ & 0.070 & 0.070 & $1.00-1.11$ & 0.043 \\
\hline \multicolumn{7}{|c|}{ Home computer ownership } \\
\hline Yes & 1.54 & $1.45-1.63$ & $<0.0001$ & $<0.0001$ & $1.40-1.60$ & $<0.0001$ \\
\hline No & 1 & - & - & - & - & - \\
\hline \multicolumn{7}{|l|}{ Internet access } \\
\hline Yes & 1.47 & $1.42-1.53$ & $<0.0001$ & $<0.0001$ & $1.23-1.35$ & $<0.0001$ \\
\hline No & 1 & - & - & - & - & - \\
\hline \multicolumn{7}{|l|}{ Family } \\
\hline \multicolumn{7}{|c|}{ Maternal level of education } \\
\hline$<9$ years & 0.76 & $0.70-0.83$ & $<0.0001$ & $<0.0001$ & $0.78-0.91$ & $<0.0001$ \\
\hline$\geq 9$ years & 1 & - & - & - & - & - \\
\hline \multicolumn{7}{|c|}{ Living with the mother } \\
\hline Yes & 1 & - & - & - & - & - \\
\hline No & 1.11 & $1.07-1.15$ & $<0.0001$ & $<0.0001$ & $1.03-1.16$ & 0.003 \\
\hline \multicolumn{7}{|c|}{ Living with the father } \\
\hline Yes & 1.51 & $1.42-1.61$ & $<0.0001$ & $<0.0001$ & $1.04-1.26$ & 0.004 \\
\hline No & 1 & - & - & - & - & - \\
\hline \multicolumn{7}{|l|}{ Behavioral } \\
\hline \multicolumn{7}{|l|}{ Use of tobacco } \\
\hline Yes & 0.67 & $0.62-0.72$ & $<0.0001$ & $<0.0001$ & $1.48-1.60$ & $<0.0001$ \\
\hline No & 1 & - & - & - & - & - \\
\hline \multicolumn{7}{|c|}{ Consumption of alcohol } \\
\hline Yes & 1 & - & - & - & - & - \\
\hline No & 0.82 & $0.79-0.85$ & $<0.0001$ & $<0.0001$ & $0.82-0.86$ & $<0.0001$ \\
\hline \multicolumn{7}{|l|}{ Physical activity } \\
\hline$\geq 3$ days a week & 1 & - & - & - & - & - \\
\hline$<3$ days a week & 1.14 & $1.09-1.20$ & $<0.0001$ & $<0.0001$ & $1.04-1.14$ & $<0.0001$ \\
\hline \multicolumn{7}{|c|}{ Meals with parents or guardians } \\
\hline$\geq 3$ days a week & 1 & - & - & - & - & - \\
\hline$<3$ days a week & 0.83 & $0.78-0.89$ & $<0.0001$ & $<0.0001$ & $0.78-0.94$ & 0.002 \\
\hline \multicolumn{7}{|c|}{ Eating while watching television or studying } \\
\hline \multicolumn{7}{|c|}{$\geq 3$ days a week } \\
\hline$<3$ days a week & & & & & & \\
\hline
\end{tabular}


Table 4. Multiple logistic regression analysis for the regular consumption of obesogenic foods among adolescents, according to sociodemographic, family, behavioral, and school structural characteristics. Brazil, 2012.

2 of 6

\begin{tabular}{|c|c|c|c|c|c|c|}
\hline \multirow{3}{*}{ Variables } & \multicolumn{6}{|c|}{ Fried snacks } \\
\hline & \multicolumn{3}{|c|}{ Crude odds Ratio } & \multicolumn{3}{|c|}{ Adjusted odds Ratio } \\
\hline & $\mathrm{OR}^{1}$ & $(95 \% \mathrm{Cl})^{2}$ & $p$ & $\mathrm{OR}^{1}$ & $(95 \% \mathrm{Cl})^{2}$ & $p$ \\
\hline \multicolumn{7}{|l|}{ Behavioral } \\
\hline \multicolumn{7}{|l|}{ Breakfast } \\
\hline$\geq 3$ days a week & 1.48 & $1.42-1.54$ & $<0.0001$ & 1.46 & $1.40-1.53$ & $<0.0001$ \\
\hline$<3$ days a week & 1 & - & - & 1 & - & - \\
\hline \multicolumn{7}{|c|}{ Time spent watching television } \\
\hline$\geq 2$ hours a day & 1 & - & - & 1 & - & - \\
\hline$<2$ hours a day & 1.26 & $1.18-1.34$ & $<0.0001$ & 1.39 & $1.31-1.48$ & $<0.0001$ \\
\hline \multicolumn{7}{|c|}{ Willingness to lose weight } \\
\hline Yes & 1 & - & - & 1 & - & - \\
\hline \multirow[t]{2}{*}{ No } & 1.26 & $1.18-1.34$ & $<0.0001$ & 1.39 & $1.31-1.48$ & $<0.0001$ \\
\hline & \multicolumn{6}{|c|}{ Packaged snacks } \\
\hline \multirow[t]{2}{*}{ Variables } & \multicolumn{3}{|c|}{ Crude odds Ratio } & \multicolumn{3}{|c|}{ Adjusted odds Ratio } \\
\hline & $\mathrm{OR}^{1}$ & $(95 \% \mathrm{Cl})^{2}$ & $p$ & $\mathrm{OR}^{1}$ & $(95 \% \mathrm{Cl})^{2}$ & $p$ \\
\hline \multicolumn{7}{|c|}{ Sociodemographic } \\
\hline \multicolumn{7}{|l|}{ Gender } \\
\hline Male & 1 & - & - & 1 & - & - \\
\hline Female & 1.27 & $1.20-1.33$ & $<0.0001$ & 1.38 & $1.33-1.44$ & $<0.0001$ \\
\hline \multicolumn{7}{|l|}{$\begin{array}{l}\text { Age } \\
\leq 14 \text { years } \\
>14 \text { years }\end{array}$} \\
\hline \multicolumn{7}{|c|}{ Currently employed } \\
\hline Yes & 1.22 & $1.16-1.27$ & $<0.0001$ & 1.19 & $1.15-1.24$ & $<0.0001$ \\
\hline No & 1 & - & - & 1 & - & - \\
\hline \multicolumn{7}{|c|}{$\begin{array}{l}\text { Household composition } \\
\leq 4 \text { persons } \\
>4 \text { persons }\end{array}$} \\
\hline \multicolumn{7}{|c|}{ Home computer ownership } \\
\hline Yes & 1.27 & $1.19-1.25$ & $<0.0001$ & 1.26 & $1.18-1.34$ & $<0.0001$ \\
\hline No & 1 & - & - & 1 & - & - \\
\hline \multicolumn{7}{|l|}{ Internet access } \\
\hline Yes & 1.25 & $1.35-1.45$ & $<0.0001$ & 1.20 & $1.12-1.29$ & $<0.0001$ \\
\hline No & 1 & - & - & 1 & - & - \\
\hline
\end{tabular}

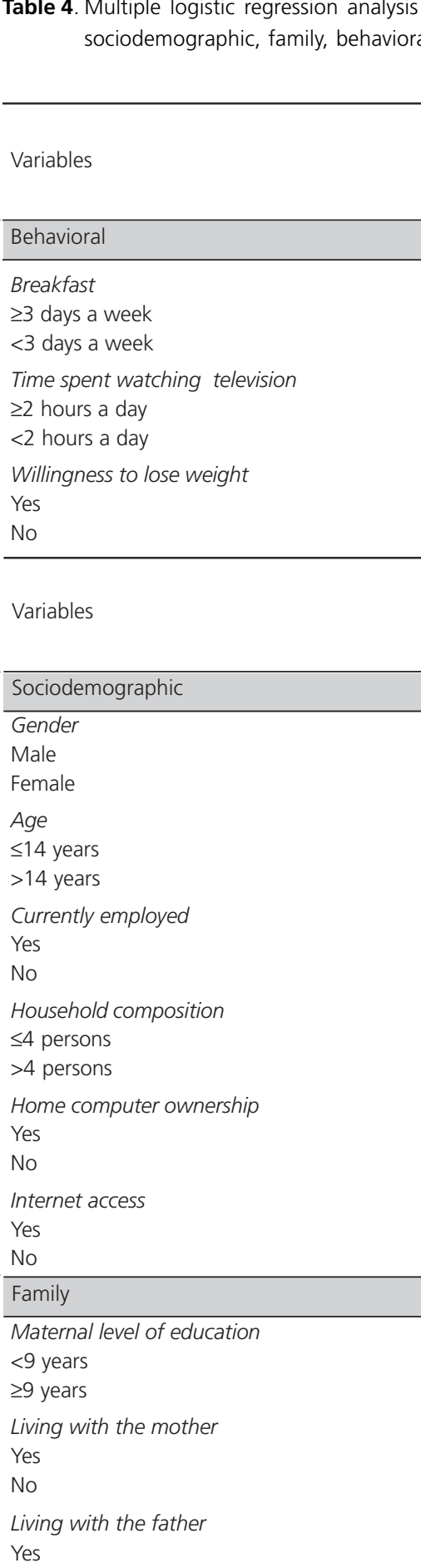

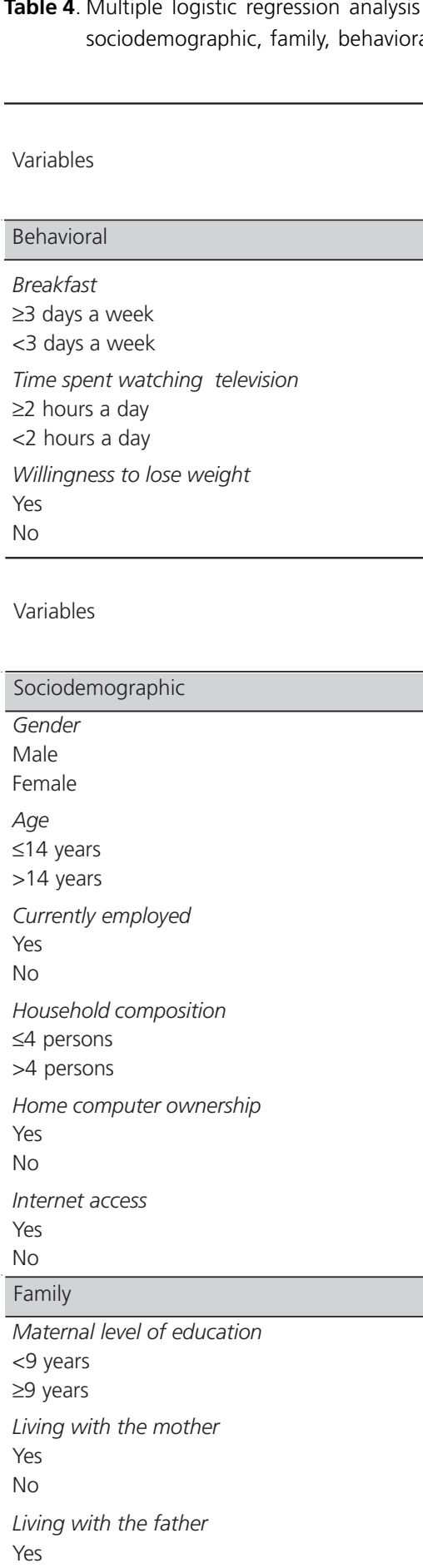

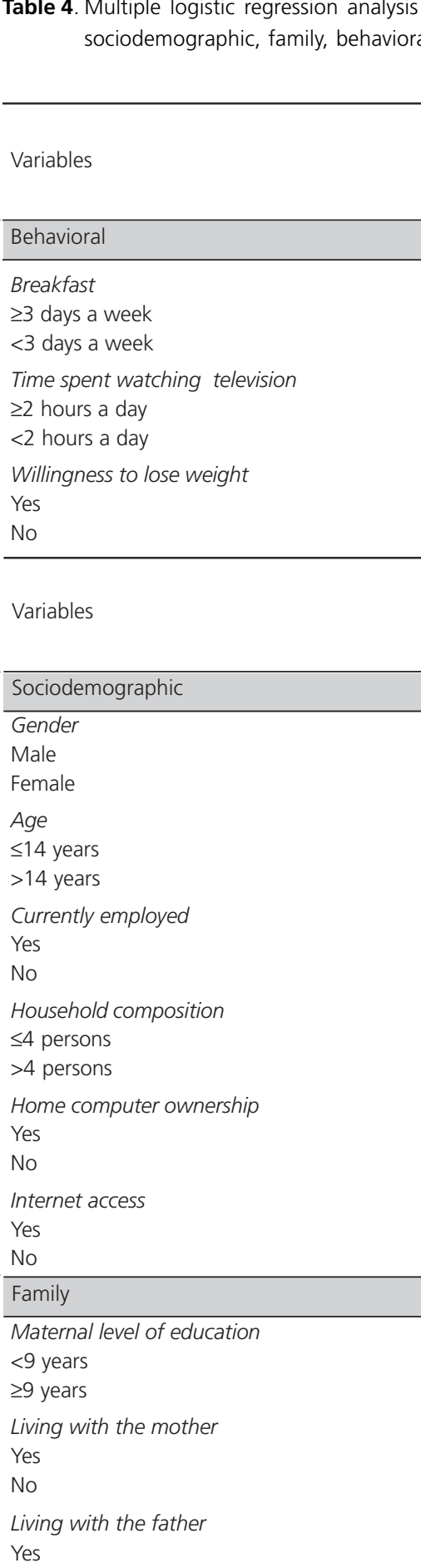

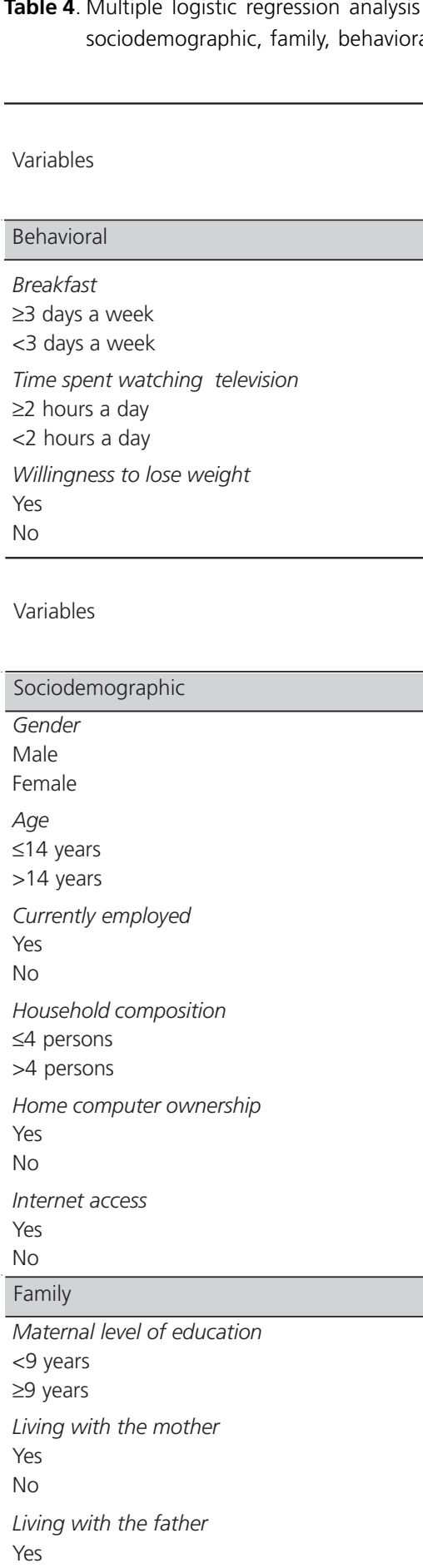

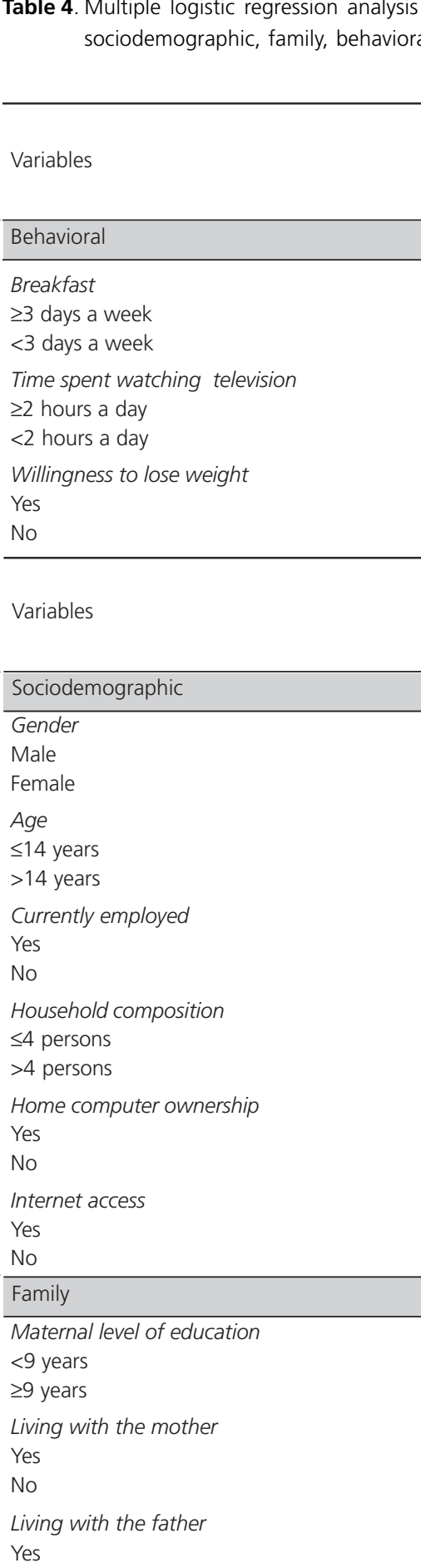

No

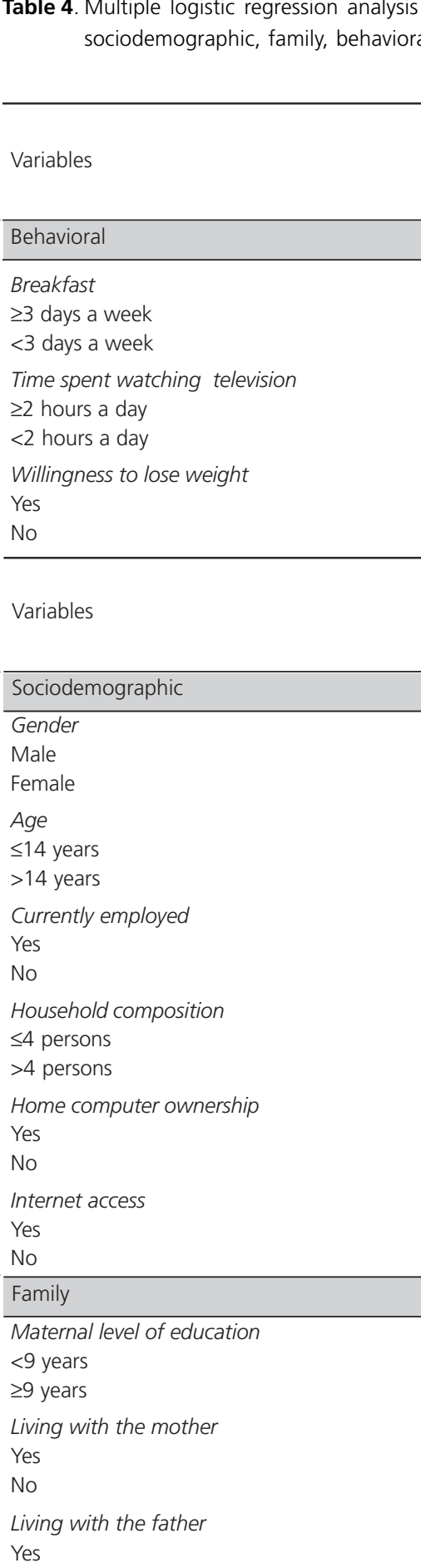

1.78

Behavioral

Use of tobacco

Yes

No

Consumption of alcohol

Yes

No

\begin{tabular}{cccccc}
1.65 & $1.53-1.77$ & $<0.0001$ & 1.52 & $1.38-1.67$ & $<0.0001$ \\
1 & - & - & 1 & - & - \\
& & & & & \\
1 & - & - & 1 & - & - \\
0.83 & $0.78-0.88$ & $<0.0001$ & 0.79 & $0.75-0.84$ & $<0.0001$ \\
\hline
\end{tabular}


Table 4. Multiple logistic regression analysis for the regular consumption of obesogenic foods among adolescents, according to sociodemographic, family, behavioral, and school structural characteristics. Brazil, 2012.

\begin{tabular}{|c|c|c|c|c|c|c|}
\hline \multirow{3}{*}{ Variables } & \multicolumn{6}{|c|}{ Packaged snacks } \\
\hline & \multicolumn{3}{|c|}{ Crude odds Ratio } & \multicolumn{3}{|c|}{ Adjusted odds Ratio } \\
\hline & $\mathrm{OR}^{1}$ & $(95 \% \mathrm{Cl})^{2}$ & $p$ & $\mathrm{OR}^{1}$ & $(95 \% \mathrm{Cl})^{2}$ & $p$ \\
\hline \multicolumn{7}{|l|}{ Behavioral } \\
\hline \multicolumn{7}{|c|}{$\begin{array}{l}\text { Physical activity } \\
\geq 3 \text { days a week } \\
<3 \text { days a week }\end{array}$} \\
\hline \multicolumn{7}{|c|}{ Meals with parents or guardians } \\
\hline$\geq 3$ days a week & 1 & - & - & 1 & - & - \\
\hline$<3$ days a week & 0.82 & $0.79-0.85$ & $<0.0001$ & 0.84 & $0.81-0.87$ & $<0.0001$ \\
\hline \multicolumn{7}{|c|}{ Eating while watching television or studying } \\
\hline$\geq 3$ days a week & 1 & - & - & 1 & - & - \\
\hline$<3$ days a week & 1.03 & $0.99-1.06$ & 0.065 & 0.96 & $0.93-0.99$ & 0.032 \\
\hline \multicolumn{7}{|l|}{ Breakfast } \\
\hline$\geq 3$ days a week & 1.57 & $1.53-1.62$ & $<0.0001$ & 1.53 & $1.47-1.58$ & $<0.0001$ \\
\hline$<3$ days a week & 1 & - & - & 1 & - & - \\
\hline \multicolumn{7}{|c|}{ Time spent watching television } \\
\hline$\geq 2$ hours a day & 1 & - & - & 1 & - & - \\
\hline$<2$ hours a day & 1.31 & $1.22-1.40$ & $<0.0001$ & 1.44 & $1.33-1.55$ & $<0.0001$ \\
\hline \multicolumn{7}{|c|}{ Willingness to lose weight } \\
\hline Yes & 1 & - & - & 1 & - & - \\
\hline \multirow[t]{2}{*}{ No } & 1.31 & $1.22-1.40$ & $<0.0001$ & 1.44 & $1.33-1.55$ & $<0.0001$ \\
\hline & \multicolumn{6}{|c|}{ Sugar candies } \\
\hline \multirow[t]{2}{*}{ Variables } & \multicolumn{3}{|c|}{ Crude odds Ratio } & \multicolumn{3}{|c|}{ Adjusted odds Ratio } \\
\hline & $\mathrm{OR}^{1}$ & $(95 \% \mathrm{Cl})^{2}$ & $p$ & $\mathrm{OR}^{1}$ & $(95 \% \mathrm{Cl})^{2}$ & $p$ \\
\hline \multicolumn{7}{|c|}{ Sociodemographic } \\
\hline \multicolumn{7}{|l|}{ Gender } \\
\hline Male & 1 & - & - & 1 & - & - \\
\hline Female & 1.62 & $1.62-1.68$ & $<0.0001$ & 1.76 & $1.70-1.82$ & $<0.0001$ \\
\hline \multicolumn{7}{|l|}{ Age } \\
\hline$\leq 14$ years & 1 & - & - & 1 & - & - \\
\hline$>14$ years & 0.76 & $0.69-0.83$ & $<0.0001$ & 0.81 & $0.76-0.85$ & $<0.0001$ \\
\hline \multicolumn{7}{|c|}{$\begin{array}{l}\text { Currently employed } \\
\text { Yes } \\
\text { No }\end{array}$} \\
\hline \multicolumn{7}{|c|}{ Household composition } \\
\hline$\leq 4$ persons & 1.32 & $1.16-1.51$ & $<0.0001$ & 1.11 & $1.05-1.17$ & $<0.0001$ \\
\hline$>4$ persons & 1 & - & - & 1 & - & - \\
\hline \multicolumn{7}{|c|}{ Home computer ownership } \\
\hline Yes & 1.34 & $1.18-1.52$ & $<0.0001$ & 1.15 & $1.06-1.25$ & $<0.0001$ \\
\hline No & 1 & - & - & 1 & - & - \\
\hline Internet access & & & & & & \\
\hline Yes & 1.44 & $1.34-1.55$ & $<0.0001$ & 1.19 & $1.14-1.25$ & $<0.0001$ \\
\hline No & 1 & - & - & 1 & - & - \\
\hline Family & & & & & & \\
\hline Maternal level of & & & & & & \\
\hline$<9$ years & 0.80 & $0.73-0.87$ & $<0.0001$ & 0.90 & $0.86-0.95$ & $<0.0001$ \\
\hline$\geq 9$ years & 1 & - & - & 1 & - & - \\
\hline Living with the $m$ & & & & & & \\
\hline Yes & & & & & & \\
\hline No & & & & & & \\
\hline
\end{tabular}


Table 4. Multiple logistic regression analysis for the regular consumption of obesogenic foods among adolescents, according to sociodemographic, family, behavioral, and school structural characteristics. Brazil, 2012.

4 of 6

\begin{tabular}{|c|c|c|c|c|c|c|}
\hline \multirow{3}{*}{ Variables } & \multicolumn{6}{|c|}{ Sugar candies } \\
\hline & \multicolumn{3}{|c|}{ Crude odds Ratio } & \multicolumn{3}{|c|}{ Adjusted odds Ratio } \\
\hline & $\mathrm{OR}^{1}$ & $(95 \% \mathrm{Cl})^{2}$ & $p$ & $\mathrm{OR}^{1}$ & $(95 \% \mathrm{Cl})^{2}$ & $p$ \\
\hline \multicolumn{7}{|l|}{ Behavioral } \\
\hline \multicolumn{7}{|l|}{ Use of tobacco } \\
\hline Yes & 1.44 & $1.35-1.54$ & $<0.0001$ & 1.22 & $1.12-1.33$ & $<0.0001$ \\
\hline No & 1 & - & - & 1 & - & - \\
\hline \multicolumn{7}{|c|}{ Consumption of alcohol } \\
\hline Yes & 1.57 & $1.48-1.67$ & $<0.0001$ & 1.52 & $1.42-1.62$ & $<0.0001$ \\
\hline No & 1 & - & - & 1 & - & - \\
\hline \multicolumn{7}{|l|}{ Physical activity } \\
\hline$\geq 3$ days a week & 1 & - & - & 1 & - & - \\
\hline$<3$ days a week & 0.83 & $0.81-0.85$ & $<0.0001$ & 0.75 & $0.73-0.77$ & $<0.0001$ \\
\hline \multicolumn{7}{|c|}{ Meals with parents or guardians } \\
\hline$\geq 3$ days a week & 1 & - & - & 1 & - & - \\
\hline$<3$ days a week & 1.18 & $1.12-1.25$ & $<0.0001$ & 1.11 & $1.06-1.15$ & $<0.0001$ \\
\hline \multicolumn{7}{|c|}{ Eating while watching television or studying } \\
\hline$\geq 3$ days a week & 1 & - & - & 1 & - & - \\
\hline$<3$ days a week & 0.91 & $0.87-0.95$ & $<0.0001$ & 0.91 & $0.88-0.94$ & $<0.0001$ \\
\hline \multicolumn{7}{|l|}{ Breakfast } \\
\hline$\geq 3$ days a week & 1 & - & - & 1 & - & - \\
\hline$<3$ days a week & 1.19 & $1.15-1.23$ & $<0.0001$ & 1.07 & $1.04-1.11$ & $<0.0001$ \\
\hline \multicolumn{7}{|c|}{ Time spent watching television } \\
\hline$\geq 2$ hours a day & 1.63 & $1.53-1.73$ & $<0.0001$ & 1.56 & $1.45-1.67$ & $<0.0001$ \\
\hline$<2$ hours a day & 1 & - & - & 1 & - & - \\
\hline \multicolumn{7}{|c|}{ Willingness to lose weight } \\
\hline Yes & 1 & - & - & 1 & - & - \\
\hline No & 1.11 & $1.07-1.15$ & $<0.0001$ & 1.30 & $1.26-1.34$ & $<0.0001$ \\
\hline \multicolumn{7}{|c|}{ School food consumption } \\
\hline$\geq 3$ days a week & 1 & - & - & 1 & - & - \\
\hline$<3$ days a week & 1.18 & $1.09-1.28$ & $<0.0001$ & 1.07 & $1.02-1.12$ & 0.005 \\
\hline \multicolumn{7}{|l|}{ School Structural } \\
\hline \multicolumn{7}{|l|}{ Cafeteria } \\
\hline Yes & 1.16 & $1.07-1.24$ & $<0.0001$ & 1.08 & $1.05-1.12$ & $<0.0001$ \\
\hline \multirow[t]{2}{*}{ No } & 1 & - & - & 1 & - & - \\
\hline & \multicolumn{6}{|c|}{ Soft drinks } \\
\hline \multirow[t]{2}{*}{ Variables } & \multicolumn{3}{|c|}{ Crude odds Ratio } & \multicolumn{3}{|c|}{ Adjusted odds Ratio } \\
\hline & $\mathrm{OR}^{1}$ & $(95 \% \mathrm{Cl})^{2}$ & $p$ & $\mathrm{OR}^{1}$ & $(95 \% \mathrm{Cl})^{2}$ & $p$ \\
\hline \multicolumn{7}{|c|}{ Sociodemographic } \\
\hline Gender & & & & & & \\
\hline Male & 1 & - & - & 1 & - & - \\
\hline Female & 0.85 & $0.81-0.88$ & $<0.0001$ & 0.89 & $0.85-0.93$ & $<0.0001$ \\
\hline Age & & & & & & \\
\hline$\geq 14$ years & & & & & & \\
\hline$>14$ years & & & & & & \\
\hline Currently employ & & & & & & \\
\hline Yes & 1.08 & $1.00-1.14$ & 0.043 & 1.36 & $1.31-1.41$ & $<0.0001$ \\
\hline No & 1 & - & - & 1 & - & - \\
\hline
\end{tabular}


Table 4. Multiple logistic regression analysis for the regular consumption of obesogenic foods among adolescents, according to sociodemographic, family, behavioral, and school structural characteristics. Brazil, 2012.

\begin{tabular}{|c|c|c|c|c|c|c|}
\hline \multirow{3}{*}{ Variables } & \multicolumn{6}{|c|}{ Soft drinks } \\
\hline & \multicolumn{3}{|c|}{ Crude odds Ratio } & \multicolumn{3}{|c|}{ Adjusted odds Ratio } \\
\hline & $\mathrm{OR}^{1}$ & $(95 \% \mathrm{Cl})^{2}$ & $p$ & $\mathrm{OR}^{1}$ & $(95 \% \mathrm{Cl})^{2}$ & $p$ \\
\hline \multicolumn{7}{|c|}{ Sociodemographic } \\
\hline \multicolumn{7}{|c|}{ Household composition } \\
\hline$\leq 4$ persons & 1.68 & $1.49-1.90$ & $<0.0001$ & 1.21 & $1.10-1.34$ & $<0.0001$ \\
\hline$>4$ persons & 1 & - & - & 1 & - & - \\
\hline \multicolumn{7}{|c|}{ Home computer ownership } \\
\hline Yes & 1.72 & $1.54-1.91$ & $<0.0001$ & 1.40 & $1.32-1.50$ & $<0.0001$ \\
\hline No & 1 & - & - & 1 & - & - \\
\hline \multicolumn{7}{|l|}{ Internet access } \\
\hline Yes & 1.48 & $1.36-1.60$ & $<0.0001$ & 1.27 & $1.19-1.35$ & $<0.0001$ \\
\hline No & 1 & - & - & 1 & - & - \\
\hline \multicolumn{7}{|l|}{ Family } \\
\hline \multicolumn{7}{|c|}{ Maternal level of education } \\
\hline$<9$ years & 0.73 & $0.67-0.80$ & $<0.0001$ & 0.89 & $0.85-0.95$ & $<0.0001$ \\
\hline$\geq 9$ years & 1 & - & - & 1 & - & - \\
\hline \multicolumn{7}{|c|}{ Living with the mother } \\
\hline Yes & 1 & - & - & 1 & - & - \\
\hline No & 1.38 & $1.29-1.48$ & $<0.0001$ & 1.05 & $0.98-1.13$ & 0.137 \\
\hline \multicolumn{7}{|l|}{ Behavioral } \\
\hline \multicolumn{7}{|l|}{ Use of tobacco } \\
\hline Yes & 1.52 & $1.38-1.68$ & $<0.0001$ & 1.22 & $1.15-1.29$ & $<0.0001$ \\
\hline No & 1 & - & - & 1 & - & - \\
\hline \multicolumn{7}{|c|}{ Consumption of alcohol } \\
\hline Yes & 1.70 & $0.63-0.76$ & $<0.0001$ & 1.59 & $1.52-1.66$ & $<0.0001$ \\
\hline No & 1 & - & - & 1 & - & - \\
\hline \multicolumn{7}{|l|}{ Physical activity } \\
\hline$\geq 3$ days a week & 1 & - & - & 1 & - & - \\
\hline$<3$ days a week & 0.82 & $0.80-0.84$ & $<0.0001$ & 0.88 & $0.85-0.92$ & $<0.0001$ \\
\hline $\begin{array}{l}\text { Meals with paren } \\
\geq 3 \text { days a week } \\
<3 \text { days a week }\end{array}$ & & & & & & \\
\hline \multicolumn{7}{|c|}{ Eating while watching television or studying } \\
\hline$\geq 3$ days a week & 1 & - & - & 1 & - & - \\
\hline$<3$ days a week & 0.83 & $0.76-0.90$ & $<0.0001$ & 0.85 & $0.82-0.88$ & $<0.0001$ \\
\hline \multicolumn{7}{|l|}{ Breakfast } \\
\hline$\geq 3$ days a week & 1 & - & - & 1 & - & - \\
\hline$<$ days a week & 1.11 & $1.07-1.15$ & $<0.0001$ & 1.07 & $1.03-1.12$ & $<0.0001$ \\
\hline \multicolumn{7}{|c|}{ Time spent watching television } \\
\hline$\geq 2$ hours a day & 1.45 & $1.40-1.49$ & $<0.0001$ & 1.44 & $1.33-1.55$ & $<0.0001$ \\
\hline$<2$ hours a day & 1 & - & - & 1 & - & - \\
\hline \multicolumn{7}{|c|}{ Willingness to lose weight } \\
\hline Yes & 1 & - & - & 1 & - & - \\
\hline No & 1.26 & $1.16-1.30$ & $<0.0001$ & 1.34 & $1.27-1.42$ & $<0.0001$ \\
\hline \multicolumn{7}{|c|}{ School food consumption } \\
\hline$\geq 3$ days a week & & & & & & \\
\hline$<3$ days a week & & & & & & \\
\hline
\end{tabular}


Table 4. Multiple logistic regression analysis for the regular consumption of obesogenic foods among adolescents, according to sociodemographic, family, behavioral, and school structural characteristics. Brazil, 2012.

6 of 6

\begin{tabular}{|c|c|c|c|c|c|c|}
\hline \multirow{3}{*}{ Variables } & \multicolumn{6}{|c|}{ Soft drinks } \\
\hline & \multicolumn{3}{|c|}{ Crude odds Ratio } & \multicolumn{3}{|c|}{ Adjusted odds Ratio } \\
\hline & $\mathrm{OR}^{1}$ & $(95 \% \mathrm{Cl})^{2}$ & $p$ & $\mathrm{OR}^{1}$ & $(95 \% \mathrm{Cl})^{2}$ & $p$ \\
\hline \multicolumn{7}{|c|}{ School Structural } \\
\hline \multicolumn{7}{|l|}{ Cafeteria } \\
\hline \multicolumn{7}{|l|}{ Yes } \\
\hline No & & & & & & \\
\hline
\end{tabular}

Note: 1 OR: Odds Ratio, calculated as weighted odds ratio due to the sampling design; ${ }^{2} 95 \%$ IC: $95 \%$ Confidence Interval, calculated as weighted confidence interval due to the sampling design.

Additionally, the adolescents that had greater probability of eating eat sugar candies regularly were females who owned a home computer and a mobile phone, had Internet access, smoked, consumed alcohol, and showed a lower frequency of eating breakfast, eating meals with the family, and school food consumption. These girls also spent more time watching television and attended schools that did not have a cafeteria.

In the final logistic model, the probability of regular consumption of soft drinks was greater among adolescents who had a job, owned a home computer and amobile phone, had Internet access, did not live with their father, smoked, consumed alcohol, ate breakfast less than 3 three days a week, watched television for more than two hours daily, and did not display willingness to lose weight.

Moreover, maternal level of education of less than nine years was a protective factor in the final adjusted models for the regular consumption of fried snacks, sugar candies, and soft drinks among the Brazilian adolescents analyzed.

\section{DISCUSSION}

The distribution of the population studied in terms of the frequency of consumption of obsegenic foods showed that their regular and daily consumption was higher among girls, except for soft drinks (difference of $0.2 \%$ between the genders). An interesting fact is that more than one third of girls reported daily consumption of sugar candies. In the logistic models used, females also showed greater probability of regular consumption of fried snacks (OR=1.20;95\% Cl 1.11 to 1.29 ), packaged snacks (OR=1:38; $95 \% \mathrm{Cl}$ 1.33 to 1.44$)$, and sugar candies $(\mathrm{OR}=1.76$; $95 \% \mathrm{Cl} 1.70-1.282)$. In general, adolescent girls were more susceptible to developing unhealthy behavior and eating habits (use of tobacco, low frequency of eating meals with parents or guardians, low-frequency physical activity, and excessive amount of time spent watching television).

Although the literature often addresses differences between genders in food consumption patterns, the results from many studies differ from those found in the present study $^{12,13}$. Investigating a representative sample of children and adolescents in the United States, some authors found that on averageboys had higher daily caloric intake, and the portion sizes and energy density of their meals and snacks were larger than those of girls ${ }^{12}$.

Yannakoulia et al..$^{13}$ evaluated the eating habits of Greek adolescents as part of a large school-based study called Health Behavior in School-Aged Children (HBSC), based on unhealthy food choicescores, among which the highest scores corresponded to the worst diet quality. The authors concluded that the girls had the lowest 
scores, afinding that was significant in all three age groups investigated (11, 13, and 15 years old).

In addition to the high percentage of adolescents who regularly consumed fried snacks, packaged snacks, sugar candies, and soft drinks with regular frequency (ranging from $27.17 \%$ to $65.96 \%$, as previously mentioned) in the final logistic regression model (after adjusting the variables), the consumption of these four types of food was statistically associated with mobile phone ownership, Internet access, watching television for more than 2 hours a day, use of tobacco, and consumption of alcohol. The low frequency (less than three days a week) of the habit of eating while watching television or studying was a protective factor $(\mathrm{OR}<1.0)$ for the regular consumption of all foods.

The grow thin mobile media use, such as mobile phones (smartphones) and tablets, has been the subject of many studies that aim to identify their impact on the lives of consumers, in particular the Generation Z (or also called Generation, cohort of people born between 1991-2000), which is the first generation born after the advent of the Internet and who are growing up in an era of global interaction ${ }^{14}$.

There is a consensus that the time spent on social networking is positively associated with the consumption of unhealthy foods, which is also consistent with research carried out by Boynton-Jarret et al. ${ }^{15}$. These authors evaluated adolescents over a period of 19 months and found an inverse relationship between the time spent watching television and the intake of fruits and vegetables. They believe that this reflects a replacement of healthy foods with unhealthy ones, which are highly advertised on television.

In addition to the negative approach associating the use of media with physical inactivity, obesity, mental disorders, reduction in sleeping hours and rest, problems with interpersonal relationships, and learning difficulties in the classroom ${ }^{16}$, these adolescents are subjected to the massive advertising for industrialized foods ${ }^{15}$.
However, given the growing trend of technological advances and their rapidly spread among children and adolescents, confronting such reality does not appear to be an effective approach. A more appropriate strategy would be investing in electronic media to promote healthy eating, advertising health enhancing-foods, and transmitting appropriate nutrition education messages ${ }^{17}$. For example, Peterson et al. ${ }^{18}$ used healthy eating videos for 20 minutes for 10 days to assess the eating habits of children aged 5 and 6 years. The results showed that the students learned nutrition concepts and displayed certain tendency towards healthy food consumption.

As for the findings related to use of tobacco and alcohol consumption, these variables, whichrefer to unhealthy life style habits, lead to harmful eating behaviors, a fact that is consistent with the literature ${ }^{19}$.

Although in Brazil the law prohibits the consumption of alcoholic beverage by any person under 18 years, the allure of alcohol is due to the fact that it is not an illegal drug and therefore is not subjected to censorship as strict as that of illegal drugs. Early age alcohol use can be related to several factors, such aseasy access and influence of friends and family. Other countries have also revealed high frequency of alcohol consumption among adolescents. It has been reported that in Coimbra, Portugal, 65\% of adolescents aged 12 to 18 have already consumed alcohol and 19\% have already gotten drunk ${ }^{20}$, and in Germany, it was found that $93 \%$ of adolescents aged 15 to 16 have already tried alcoholic berverages ${ }^{21}$.

Moreover, based on data from the Project Eating Among Teens (EAT), a study designed to investigate socio environmental, personal, and behavioral factors associated with dietary intake in adolescents 11-18 years, Larson et al. ${ }^{19}$ reported results regarding smoking and factors associated with it. The authors concluded that smoking among adolescents was inversely related to the frequency of meals (breakfast, lunch, and 
dinner) and consumption of fruits, vegetables, grains, and some vitamins and minerals. Smoking frequency was directly related to the frequency of fast-food and soft drink consumption.

The results from the present study revealed that the like lihood that adolescents will consume fried snacks, packaged snacks, sugar candies, and soft drinks was higher among those who worked. Considering that all adolescents were attending schools, such association may reflect the fact that they had less time to have regular meals and frequently ate outside the home. Furthermore, given the characteristics of these food groups, it can be said that these preparations and foods have been replacing the major meals. Corroborating this assertion, a study found that one third of adolescents replaced lunch and half of them replaced dinner with snacks, a habit that was most common among girls. Sandwiches, savory snacks, pizzas, and hamburgers were the main meal replacements ${ }^{22}$.

Similarly, our study found that adolescents who had breakfast less than three days a week were more likely to regularly consume packaged snacks, sugar candies, and soft drinks.

Skipping breakfast is common among adolescents and has been associated with higher body mass index, and attention and concentration problems, with possible impacts on adult health. A longitudinal study conducted in Australia showed that both children and adults who skipped breakfast had larger waist circumference, higher fasting insulin, and higher levels of total cholesterol and Low Density Lipoprotein cholesterol in adulthood when compared to those who ate breakfast during childhood and adulthood $^{23}$. One reason for this finding is the association of this habit with other unhealthy lifestyle habits, such as low fruit and vegetable intake.

Within the same context, in a prospective study of adolescents participating in the National Longitudinal Study of Adolescent Health, Niemeier et al..$^{24}$ investigated whether fast food consumption and breakfast skipping were associated with weight gain during the transition from adolescence to adulthood. The authors found marked increases in the consumption of these foods and decreases in the consumption of breakfast for five years. Another important finding is that these dietary behaviors were associated with increased weight gain from adolescence to adulthood.

With regard to the role of the family in the eating habits of adolescents, our findings revealed a significant association between low frequency of meals with parents or guardians (less than three days a week) and regular consumption of sugar candies and soft drinks. This variable remained in the explanatory model proposed after final adjustment.

An interesting finding was that low maternal level of education (less than 9 years) was as a protective factor $(O R<1: 00)$ for the consumption of fried snacks, sugar candies, and soft drinks since the regular consumption of these types of foods is frequently found among the children with mothers that have higher levels of education. Similarly, investigating the association between maternal determinants and dietary patterns of children, Villa et al..$^{25}$ found that children whose mothers had nine or more years of education consumed more "sugary drinks and snacks". These authors also showed that the probability of children having a diet rich in these food items increased by four times when maternal level of education was 12 or more years, compared to mothers who had less years of education.

However, these data challenge the assumption that higher level of education means greater access to health-related information and greater ability to distinguish between what is healthy and what is not. Thus, contrary to our results, a cross-sectional population study carried out in Spain, found that children whose mothers had lower level of education were more likely to follow the eating pattern called "Snacky", characterized by increased consumption of bakery products, salted snacks, sweets, and soft drinks ${ }^{26}$. 
Furthermore, the adolescents who did not reported willingness to lose weight (70\%) had the highest frequency of consumption of all obesogenic foods investigated $(p<0.05)$, and this variable remained in all final explanatory models after final adjustment of the other variables. This fact may indicate that body weight dissatisfaction among adolescents leads to behavior changes and reduced unhealthy food consumption to achieve weight loss.

It is known that during adolescence there are changes in anthropometric measures and body composition, and individuals acquire approximately $50 \%$ of their final weight and $20 \%$ of their final height. These common changes during puberty often trigger body dissatisfaction associated with the influence of psychological factors (search for a new identity and social and emotional independence), peers, friends, family, and the media. Therefore, the search for a thin body as a necessary attribute to achieve success, happiness, and beauty, results in increased body dissatisfaction and larger desire to lose weight. These facts consequently lead to dietary restrictions or compensatory behaviors to prevent weight gain, which are predictors of many eating disorders ${ }^{27}$. Although their mechanisms have not yet been defined, the literature has associated eating disorders, especially anorexia and bulimia, with lipid disorders and metabolic syndrome. It has been reported that these disorders occur due to physiological and hormonal changes and also by excessive intake of unhealthy foods followed by compensatory behaviors, typical of some eating disorders ${ }^{28}$.

Finally, considering that PeNSE was conducted in the school context, it is worth highlighting that the regular consumption of the foods studied was higher among the students who attended schools that had a cafeteria and consumed school food less than three days a week. However, after adjustment in the multivariate analysis, there was no significant association between having a cafeteria and consuming school food with the consumption of fried snacks, packaged snacks, and soft drinks. In the final explanatory model, the variables remained associated only with the regular consumption of sugar candies among teenagers.

It is important to mention that although there are initiatives to improve school cafeteria food items, high energy density foods, which are rich in sugar, fat, and salt, such as fried snacks, packaged snacks, sugar candies, and soft drinks, are still available, indicating students' preference forthem ${ }^{29}$. This is due to the high prevalence of outsourced management of school cafeterias, with little interference from the school community, and thus the owner has autonomy and focuses primarily on profit generation at the expense of a healthy diet. Therefore, improving the nutritional quality of foods sold around schools should be a continuous integration effort among suppliers, school board, students, families, and the governement 29 .

With regard to the consequences of the type of foods available in schools and school surroundings, Virtanen et al. ${ }^{30}$ investigated the association among the proximity of fast food chains and grocery stores to schools and the food choices of Finnish adolescents. The authors found an association between the presence of these food establishments in the vicinity of schools and poor eating habits, such as skipping breakfast, low consumption of free school meals, and a 1.25-fold risk of overweight.

After discussing the factors associated with the regular consumption of obesogenic foods considering literature data and trying to understand these associations, it is important to add that there is a consensus in the literature on the negative consequences of this behavior ${ }^{5}$.

In addition to the exponentially increased risk of overweight and obesity, the consumption of fried snacks, packaged snacks, sugar candies, and soft drinks contributes to Non Communicable Diseases (NCD). The incidence of NCD has increased in the past decades among adolescents ${ }^{31}$. These foods have high energy density, high sugar content, high levels of saturated fat and trans fat, 
and are low in fibers ${ }^{3}$, which affects the body's ability to maintain energy balance, increasing the risk of gaining too much weight, prevalence of dental caries, and morbidity and mortality due to cardiovascular disease, hypertension, diabetes, and several forms of cancers ${ }^{5}$.

It should be added that, as demonstrated by Johnson \& Kenny ${ }^{32}$, like other substances (such as cocaine or heroin), unhealthy foods can be addictive. This is due to the fact that their consumption triggers progressive deterioration of the chemical balance in brain reward circuitries leading to the development of a pattern of compulsive eating, especially foods that are high in fats and calories, contributing to the maintenance of these food preferences throughout the life span.

The concept of tracking has been defined in epidemiology as the stability of a given variable over a period of time. Dietary tracking values can therefore be considered to illustrate the maintenance of dietary habits, nutrient intakes, or food consumption over time ${ }^{33}$. There is a tendency to change(or absence of 'tracking') in these standards, which appear to be negative, i.e., there is a decrease in dietary quality throughout the life span.

Based on data from the Framingham Children's study, which evaluated children aged 3 to 5 years over a span of up to 6 years six years, Singer et al. ${ }^{34}$ showed a prevalence of tracking of $40-90 \%$ both in the highest and the lowest quintiles of intake, and $57 \%$ of the individuals remained in the highest quintile of fat intake for three years. The authors considered tracking as the proportion of children in the highest and lowest quintiles of nutrient density intake at baseline who remained in the same quintile or moved only one quintile in subsequent years. The study supports the idea that the levels of intake of nutrients are maintained in subsequent ages.

One limitation of our study is the crosssectional design, which prevents cause-and-effect inferences. Therefore, the findings should be treated with caution until a longitudinal assessment of risk factors is carried out. Although cross-sectional studies do not enable causal inferences, they are essential for generating hypotheses and planning prospective studies that can establish clear relationships between the determinants of overweight among adolescents. Despite this limitation, the sampled population in the present study was representative of the of Brazilian schoolchildren, allowing the conclusion that the regular consumption of obesogenic foods is a major eating-related problem among this population.

\section{CONCLUSION}

Considering the objective of this study, it can be said that the consumption of fried snacks, packaged snacks, sugar candies, and soft drinks is regular in the diet of Brazilian adolescents. The consumption of these foods is associated with sociodemographic, family, behavioral, and school structural variables. It is worth highlighting that the variables female gender, mobile phone ownership, Internet access at home, use of tobacco, alcohol consumption, regular physical activity, eating while watching television or studying, watching television for at least 2 hours a day, and not willing to lose weight were associated with regular consumption in the final explanatory models of the four types of foods analyzed.

We hope our findings may lead to a new and better understanding of the profile of adolescents who regularly consume unhealthy and obesogenic foods. The identification of the factors that are associated with the consumption of these foods shows that strategies that promote healthy eating should consider sociodemographic, family, behavioral, and environmental characteristics.

Therefore, interventions should be designed aiming at a health promoting school, including, in a systematic way, the topic 'eating' into the regular school curriculum. These strategies must also be associated with the 
availability of healthy food sand the restriction of the sale of obesogenic foods in schools and their surroundings.

\section{ONTRIBUTORS}

G LONGO-SILVA contributed to the conception and design of this study, data processing, analysis and interpretation, and manuscript writing. RCE MENEZES contributed to the conception and design of this study, data processing, analysis and interpretation, manuscript writing, and critical revision of the final version. CAN SOUZA contributed to the data analysis and interpretation and manuscript writing. PM MARINHO contributed to the data analysis and interpretation. MHA TOLONI contributed to the manuscript writing and revision. MAA OLIVEIRA contributed to the critical revision of the final version of the manuscript.

\section{REFERENCES}

1. Powell P, Spears K, Rebori M. What is obesogenic environment? University of Nevada Cooperative Extension. Fact Sheet-10-11. 2010 [cited 2015 Aug 27]. Available from: http://www.unce.unr.edu/ publications/files/hn/2010/fs1011.pdf

2. Lin BH, Frazao E. Nutritional quality of foods at and away from home. Food Rev 1997 [cited 2015 Aug 19]; 20:33-40. Available from: http:// www.ers.usda. gov/media/977761/eib-105.pdf

3. Moubarac JC, Parra DC, Cannon G, Monteiro CA. Food classification systems based on food processing: Significance and implications for policies and actions: A systematic literature review and assessment. Curr Obes Rep. 2014; 3(2):256-72. http://dx.doi.org/10.1007/s13679-014-0092-0

4. Ogden CL, Carroll MD, Kit BK, Flegal KM. Prevalence of childhood and adult obesity in the United States. 2011-2012. Jama. 2014; 311(8):806-4. http://dx.doi.org/10.1001/jama.2014.732

5. Tsiros MD, Coates AM, Howe PR, Grimshaw PN, Buckley JD. Obesity: The new childhood disability? Obes Rev. 2011; 12:26-36. http://dx.doi.org/10. 1111/j.1467-789X.2009.00706.x

6. Story M, Neumark-Sztainer D, French S. Individual and environmental influences on adolescent eating behaviors. J Am Diet Assoc. 2002; 102(Suppl. 3):S40-51. http://dx.doi.org/10.1016/S0002-82 23(02)90421-9
7. Schmidt M, Affenito SG, Striegel-Moore R, Khoury PR, Barton B, Crawford P, et al. Fast food intake and diet quality in black and white girls: The National Heart, Lung, and Blood Institute Growth and Health Study. Arch Pediatr Adolesc Med. 2005; 159(7):626-31. http://dx.doi.org/10.1001/ archpedi.159.7.626

8. Martins APB, Levy RB, Claro RM, Moubarac JC, Monteiro CA. Participação crescente de produtos ultraprocessados na dieta brasileira (1987-2009). Rev Saúde Públ. 2013; 47(4):656-65. http://dx. doi.org/10.1590/S0034-8910.2013047004968

9. Currie C, Roberts C, Morgan A, Smith R, Settertobulte W, Samdal O, et al. Young people's health in context. Health Behaviour in School-aged Children (HBSC) study: International report from the 2001/2002 survey. Health Policy for Children and Adolescents, $\mathrm{N}^{\circ}$ 4. Copenhagen: WHO Regional Office for Europe; 2004 [cited 2016 July 26]. Available from: http://www.tokb.ru/elibrary/ book/med/e82923_part_1.pdf

10. Vinholes DB, Assuncao MC, Neutzling MB. Frequency of healthy eating habits measured by the 10 Steps to Healthy Eating score proposed by the Ministry of Health: Pelotas, Rio Grande do Sul State, Brazil. Cad Saúde Pública 2009; 25(4):791-9. http://dx.doi.org/10.1590/S0102-311X20090004 00010

11. Couto SF, Madruga SW, Neutzling MB, Silva MC. Frequência de adesão aos "10 Passos para uma alimentação saudável" em escolares adolescentes. Ciênc Saúde Coletiva. 2014; 19(5):1589-99. http:// dx.doi.org/10.1590/1413-81232014195.21392 013

12. Huang TT, Howarth NC, Lin BH, Roberts SB, McCrory MA. Energy intake and meal portions: Associations with BMI percentile in U.S. children. Obes Res. 2004; 12(11):1875-85. http://dx.doi.org/ 10.1038/oby.2004.233

13. Yannakoulia M, Karayiannis D, Terzidou M, Kokkevi A, Sidossis LS. Nutritionrelated habits of Greek adolescents. Eur J Clin Nutr. 2004; 58:580-6. http:// dx.doi.org/10.1038/sj.ejcn.1601849

14. Tapscott D. Grown Up Digital: How the net generation is changing our world. New York: McGraw-Hill; 2009.

15. Boynton-Jarrett R, Thomas TN, Peterson KE, Wiecha J, Sobol AM, Gortmaker SL. Impact of television viewing patterns on fruit and vegetable consumption among adolescents. Pediatrics. 2003; 112(6):1321-6. http://dx.doi.org/10.1542/peds. 112.6.1321

16. Tsitsika A, Janikian M, Schoenmakers TM, Tzavela EC, Olafsson K, Wójcik S, et al. Internet addictive behavior in adolescence: A cross-sectional study in 
seven european countries. Cyberpsychol Behav Soc Netw. 2014; 17(8):528-35. http://dx.doi.org/ 10.1089/cyber.2013.0382

17. Jones KDNP, Eathington PDNP, Baldwin KRN, Sipsma $H$. The impact of health education transmitted via social media or text messaging on adolescent and young adult risky sexual behavior: A systematic review of the literature. Sex Transm Dis. 2014; 41(7):413-9. http://dx.doi.org/10.1097/OLQ.0000 000000000146

18. Peterson PE, Jeffrey DB, Bridgwater CA, Dawson B. How pronutrition television programming affects children's dietary habits. Dev Psychol. 1983; 20(1):55-63. http://dx.doi.org/10.1037/0012-16 49.20.1.55

19. Larson NI, Story M, Neumark-Sztainer D, Hannan PJ, Perry CL. Are diet and physical activity patterns related to cigarette smoking in adolescents? Findings from Project EAT. Prev Chronic Dis. 2007 [cited 2015 Aug 18]; 4(3):A51. Available from: http://www.ncbi.nlm.nih.gov/pmc/articles/PMC19 55390/pdf/PCD43A51.pdf

20. Barroso T, Mendes A, Barbosa A. Analysis of the alcohol consumption phenomenon among adolescents: Study carried out with adolescents in intermediate public education. Rev Latino-Am Enfermagem. 2009; 17(3):347-53. http://dx.doi. org/10.1590/S0104-11692009000300011

21. Stolle M, Sack PM, Thomasius R. Binge drinking in childhood and adolescence: Epidemiology, consequences, and interventions. Dtsch Arztebl Int. 2009; 106(19):323-8. http://dx.doi.org/10.3238/ arztebl.2009.0323

22. Teixeira AS, Philippi ST, Leal GVS, Araki EL, Estima CCP, Guerreiro RER. Substituição de refeições por lanches em adolescentes. Rev Paul Pediatr. 2012; 30(3):330-7. http://dx.doi.org/10.1590/S0103-058 22012000300005

23. Smith KJ, Gall SL, McNaughton SA, Blizzard L, Dwyer T, Venn AJ. Skipping breakfast: Longitudinal associations with cardiometabolic risk factors in the childhood determinants of adult health study. Am J Clin Nutr. 2010; 92(6):1316-25. http://dx.doi.org/ 10.3945/ajcn.2010.30101

24. Niemeir HM, Raynor HÁ, Lloyd-Richardson EE, Rogers ML, Wing RR. Fast food consumption and breakfast skipping: Predictors of weight gain from adolescence to adulthood in a nationally representative sample. J Adolesc Health. 2006; 39(6):842-49. http://dx.doi.org/10.1016/j. jadohealth.2006.07.001

25. Villa JKD, Silva AR, Santos TSS, Ribeiro AQ, Pessoa MC, Sant'Ana LFR. Padrões alimentares de crianças e determinantes socioeconômicos, comportamentais e maternos. Rev Paul Pediatr. 2015; 33(3)302-9. http://dx.doi.org/10.1016/j.rpped.2015.05.001
26. Aranceta J, Pérez-Rodrigo C, Ribas L, Serra-Majem L. Sociodemographic and lifestyle determinants of food patterns in Spanish children and adolescents: The enKid study. Eur J Clin Nutr. 2003; 57(Suppl. 1):S40-4. http://dx.doi.org/10.1038/sj.ejcn.16018 13

27. Fortes LS, Meireles JFF, Neves CM, Almeida SS, Ferreira MEC. Disordered eating, body dissatisfaction, perfectionism, and mood state in female adolescentes. Rev Nutr. 2015; 28(4):371-83. http://dx.doi.org/10. 1590/1415-52732015000400004

28. Monteleone $P$, Santonastaso $P$, Pannuto $M$, Favaro A, Caregaro L, Castaldo E, et al. Enhanced serum cholesterol and triglyceride levels in bulimia nervosa: Relationships to psychiatric comorbidity, psychopathology and hormonal variables. Psychiatry Res. 2005; 134(3):267-73. http://dx.doi.org/10.10 16/j.psychres.2004.06.019

29. Porto EBS, Schmitz AS, Recine E, Rodrigues MLCF. School canteens in the Federal District, Brazil and the promotion of healthy eating. Rev Nutr. 2015; 28(1):29-41. http://dx.doi.org/10.1590/1415-5273 2015000100003

30. Virtanen M, Kivimãki H, Ervasti J, Oksanen T, Pentti J, Kouvonen A, et al. Fast food outlets and grocery stores near school and adolescents' eating habits and overweight in Finland. Eur J Public Health. 2015; 25(4):650-5. http://dx.doi.org/10.1093/ eurpub/ckv045

31. Malta DC, Andreazzi MAR, Oliveira-Campos M, Andrade SSCA, Sá NNB, Loura L, et al. Tendência dos fatores de risco e proteção de doenças crônicas não transmissíveis em adolescentes, Pesquisa Nacional de Saúde do Escolar (PeNSE 2009 e 2012). Rev Bras Epidemiol. 2014; 17(Suppl. 1):77-91. http:// dx.doi.org/10.1590/1809-4503201400050007

32. Johnson PM, Kenny PJ. Dopamine D2 receptors in addiction-like reward dysfunction and compulsive eating in obese rats. Nat Neurosci. 2010; 13(5):635-41. http://dx.doi.org/10.1038/nn.2519

33. Mikkila V, Rasanen L, Raitakari OT, Pietinen P, Viikari J. Consistent dietary patterns identified from childhood to adulthood: The cardiovascular risk in Young Finns Study. Br J Nutr. 2005; 93(6):923-31. http://dx.doi.org/10.1079/BJN20051418

34. Singer MR, Moore LL, Garrahie EJ, Ellison RC. The tracking of nutrient intake in young children: The framingham children's study. Am J Public Health. 1995 [cited 2016 July 26]; 85(12):1673-7. Available from: http://www.ncbi.nlm.nih.gov/pmc/articles/ PMC 1615722/pdf/amjph00450-0075.pdf

Received: September 21, 2015 Final version: March 3, 2016 Approved: July 14, 2016 
